Supporting Information for

\title{
Crystalline and Stable Benzofuran-Linked Covalent Organic Frameworks from
}

\section{Irreversible Cascade Reactions}

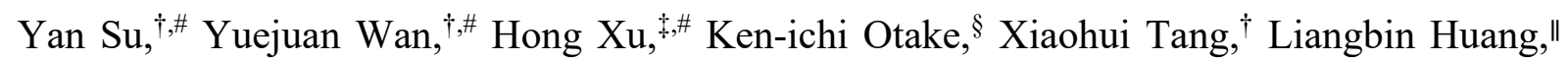
Susumu Kitagawa, ${ }^{\S}$ and Cheng $\mathrm{Gu}^{*, \dagger}$

${ }^{+}$State Key Laboratory of Luminescent Materials and Devices, Institute of Polymer Optoelectronic Materials and Devices, South China University of Technology. Guangzhou 510640, P. R. China

${ }^{\ddagger}$ Institute of Nuclear and New Energy Technology, Tsinghua University. Beijing, 100084, P. R. China

${ }^{\S}$ Institute for Integrated Cell-Material Sciences, Institute for Advanced Study, Kyoto University. Kyoto 606-8501, Japan

"School of Chemistry and Chemical Engineering, South China University of Technology. Guangzhou 510641, P. R. China

\section{Table of Contents}

Supporting Materials and Methods ............................................ 2

Supporting Tables ............................................................. 6

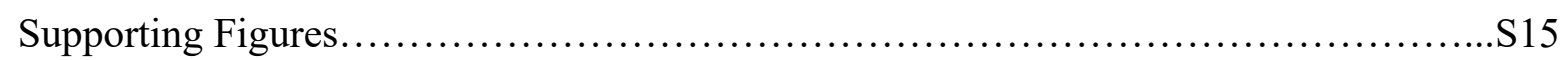

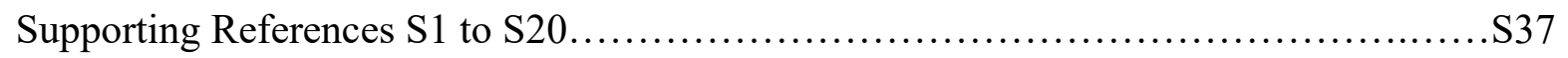




\section{Supplementary Materials and Methods}

\section{Materials and synthetic procedures}

2,5-Dihydroxyterephthalaldehyde, 2,2',2"-(benzene-1,3,5-triyl)triacetonitrile, 2,4,6trihydroxybenzene-1,3,5-tricarbaldehyde, 2,2'-(1,4-phenylene)diacetonitrile, 2phenylacetonitrile, potassium tert-butoxide, $N, N$-dimethylformamide (DMF), tetrahydrofuran (THF), mesitylene, methanol, acetone, and ethanol were purchased from J\&K Chemicals. DMSO- $d_{6}$ and $\mathrm{CDCl}_{3}$ were purchased from TCI. All the chemicals were used directly without further purification.

\section{Synthesis of 2,6-diphenylbenzo[1,2-b:4,5-b']difuran-3,7-dicarbonitrile (1). 2,5-}

Dihydroxyterephthalaldehyde ( $83 \mathrm{mg}, 0.5 \mathrm{mmol})$, 2-phenylacetonitrile (175.5 mg, $1.5 \mathrm{mmol}$ ), potassium tert-butoxide (224.4 mg, 4 equiv.), and DMF $(5 \mathrm{~mL})$ were placed in a balloonlinked $20 \mathrm{~mL}$ two-neck flask whose inner gas was replaced by $\mathrm{O}_{2}$. The mixture was stirred at $105^{\circ} \mathrm{C}$ for $24 \mathrm{~h}$. After cooling down to room temperature, the reaction was quenched by adding $10 \mathrm{~mL}$ water and filtered. The solids were dissolved in DCM and washed with saturated $\mathrm{NaCl}$ solution. The organic layer was concentrated under reduced pressure. The product was isolated through a silica gel column eluted with petroleum (PE)/ethyl acetate (EA), and then dried in vacuum at $80{ }^{\circ} \mathrm{C}$ to give 1 as a white solid in $84 \%$ isolated yield. ${ }^{1} \mathrm{H}$ NMR (500 MHz, $\left.\mathrm{CDCl}_{3}\right): \delta(\mathrm{ppm})=7.56-7.61(\mathrm{~m}, 6 \mathrm{H}), 7.89(\mathrm{~s}, 2 \mathrm{H}), 8.25(\mathrm{dd}, J=5.0,5.0 \mathrm{~Hz}$, $4 \mathrm{H}) .{ }^{13} \mathrm{C} \mathrm{NMR}\left(126 \mathrm{MHz}, \mathrm{CDCl}_{3}\right): \delta(\mathrm{ppm})=163.42,150.95,131.80,129.39,127.53,126.75$, 113.94, 102.20, 88.43. APCI MS: calcd. for $[\mathrm{M}]^{+}, \mathrm{m} / \mathrm{z}=360.37$; found $\mathrm{m} / \mathrm{z}=360.0$. The structure of 1 was also determined by SCXRD data.

Synthesis of GS-COF-1 and -2. We screened various synthetic conditions including solvents, temperature and reaction time (Table S1). Under optimal conditions for GS-COF-1 synthesis, a mixture of 2,5-dihydroxyterephthalaldehyde (16.6 mg, $0.1 \mathrm{mmol}), 2,2$ ',2"-(benzene-1,3,5triyl)triacetonitrile $(13.0 \mathrm{mg}, 0.066 \mathrm{mmol})$, potassium tert-butoxide $(44.9 \mathrm{mg}, 0.4 \mathrm{mmol})$ and DMF $(2 \mathrm{~mL})$ were placed in a $10 \mathrm{~mL}$ glass vial. The vial was charged with oxygen by flowing the oxygen gas for $5 \mathrm{~min}$. Then the vial was sealed by screwing the cover tightly and heated at $105{ }^{\circ} \mathrm{C}$ for $6 \mathrm{~d}$. Under optimal conditions for GS-COF-2 synthesis, a mixture of 2,4,6trihydroxybenzene-1,3,5-tricarbaldehyde $\quad(15.8 \quad \mathrm{mg}, \quad 0.075 \quad \mathrm{mmol}), \quad 2,2^{\prime}-(1,4-$ phenylene)diacetonitrile $(17.8 \mathrm{mg}, 0.113 \mathrm{mmol})$, potassium tert-butoxide $(33.7 \mathrm{mg}, 0.3 \mathrm{mmol})$ and DMF $(2 \mathrm{~mL})$ were placed in a $10 \mathrm{~mL}$ glass vial. The vial was charged with oxygen by 
flowing the oxygen gas for $5 \mathrm{~min}$. Then the vial was sealed by screwing the cover tightly and heated at $105{ }^{\circ} \mathrm{C}$ for $6 \mathrm{~d}$. The resulting precipitates were collected by centrifugation, subjected to Soxhlet extraction with anhydrous dichloromethane, methanol and THF for $24 \mathrm{~h}$ for each solvent, and then was dried to produce the GS-COF-1 and -2 in $88 \%$ and $92 \%$ isolated yields, respectively.

Synthesis of GS-COF-1-COOH and GS-COF-2-COOH. To a solution of $3 \mathrm{~mL}$ concentrated $\mathrm{H}_{2} \mathrm{SO}_{4}, 3 \mathrm{~mL} \mathrm{H} \mathrm{H}_{2} \mathrm{O}$ and $1 \mathrm{~mL}$ glacial acetic acid was added $100 \mathrm{mg}$ of GS-COF-1 or -2 . The suspension was stirred at $105{ }^{\circ} \mathrm{C}$ for $48 \mathrm{~h}$. Upon cooling, the mixture was diluted with deionized water $(50 \mathrm{~mL})$, and the dark precipitate was collected by filtration, rinsed with water and methanol, and dried under high vaccum to give GS-COF-1-COOH and GS-COF-2$\mathrm{COOH}$.

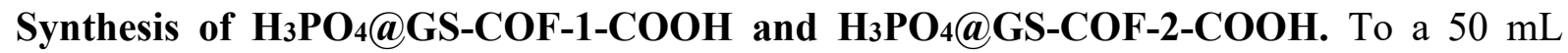
one-neck flask containing $25 \mathrm{~mL}$ of $12 \mathrm{M} \mathrm{H}_{3} \mathrm{PO}_{4}$ was added $100 \mathrm{mg}$ of GS-COF-1-COOH or GS-COF-2-COOH. The suspension was stirred at $60{ }^{\circ} \mathrm{C}$ for $4 \mathrm{~h}$. The solid was then filtered, washed with water $(50 \mathrm{~mL} \times 3)$, and vacuum dried at $120{ }^{\circ} \mathrm{C}$ for $2 \mathrm{~d}$ to give $\mathrm{H}_{3} \mathrm{PO}_{4} @ \mathrm{GS}$ COF-1-COOH and $\mathrm{H}_{3} \mathrm{PO}_{4} @$ GS-COF-2-COOH.

\section{Methods.}

${ }^{1} \mathrm{H}$ and ${ }^{13} \mathrm{C}$ NMR spectra were recorded on a Bruker AVANCE HD III 500M NMR spectrometer, where the chemical shifts ( $\delta$ in $\mathrm{ppm})$ were determined with respect to tetramethylsilane (TMS) as an internal reference. ${ }^{13} \mathrm{C}$ CP-MAS NMR spectra were recorded on a Bruker AVANCE HD III 500M NMR spectrometer. Mass spectrometry data of compounds were obtained on a Waters ACQUITY TQD liquid chromatograph-mass spectrometer using APCI ionization. Elemental analysis was performed on an Elementar Vario EL elemental analyser. Fourier transform Infrared (FT-IR) spectra were recorded on an IFS 66V/S Fourier transform infrared spectrophotometer. Single-crystal X-ray diffraction (SXRD) measurements were performed on a Rigaku XtaLAB P200 diffractometer equipped with a Dectoris PILATUS $200 \mathrm{~K}$ detector, using a VariMax Mo Optic with Mo-Ka radiation $(\lambda=0.71075 \AA)$. The structure was solved using direct methods and refined by full-matrix least-squares cycles in SHELX 2014/7. ${ }^{\mathrm{S} 1}$ All non-hydrogen atoms were refined using anisotropic thermal parameters. Powder X-ray diffraction (PXRD) measurements were performed on a Rigaku SmartLab X-ray diffractometer using $\mathrm{Cu}-\mathrm{K} \alpha$ radiation $(\lambda=1.54178$ 
$\AA$ ) in the $2 \theta$ range of $3-40^{\circ}$ with a scanning rate of $5^{\circ} \mathrm{min}^{-1}$. TGA measurements were performed on a Rigaku Thermo plus EVO2 under $\mathrm{N}_{2}$, by heating to $800{ }^{\circ} \mathrm{C}$ at a rate of $5{ }^{\circ} \mathrm{C}$ $\min ^{-1}$. Field-emission scanning electron microscopy (FE-SEM) was performed on a Zeiss_Merlin operating at an accelerating voltage of $5.0 \mathrm{kV}$. The sample was prepared by drop-casting an ethanol solution onto mica substrate followed by thermal evaporation and then coated with platinum. High-resolution transmission electron microscopy (HR-TEM) images were obtained on a TEM JEOL $2100 \mathrm{~F}$ with an acceleration voltage of $300 \mathrm{kV}$.

\section{Theoretical simulation.}

The crystalline structures of COFs were calculated using density-functional theory ${ }^{\mathrm{S} 2 \mathrm{~S} 3}$ (DFT) implemented in the $\mathrm{CASTEP}^{\mathrm{S} 4}$ module of Materials Studio 7.0. The generalized gradient approximation (GGA) in the form of Perdew-Burke-Ernzerhof (PBE) ${ }^{\mathrm{S} 5}$ was selected as the exchange-correlation functional. Grimme dispersion correction ${ }^{\mathrm{S} 6, \mathrm{~S} 7}$ was employed in all calculations to describe van der Waals $(\mathrm{vdW})$ and $\pi$-stacking interactions. The lattice dimensions were optimized simultaneously with the geometry. A plane wave energy cutoff of $830 \mathrm{eV}$ and the Monkhorst-Pack $k$-point grid of $1 \times 1 \times 4$ were used. The convergence criteria for energy, force, stress and displacement are $5 \times 10^{-6} \mathrm{eV} /$ atom, $0.01 \mathrm{eV} / \AA \AA, 0.02 \mathrm{GPa}$ and $5 \times$ $10^{-4} \AA$, respectively. All simulation works were performed using the computing resources at National Supercomputing Center in Shenzhen.

\section{Gas sorption measurements.}

Gas sorption isotherms were measured on a BELSORP-mini (MicrotracBEL, Japan, Corp.) automated volumetric sorption analyser. Before measurement, the samples were degassed in vacuum at $120{ }^{\circ} \mathrm{C}$ for $11 \mathrm{~h}$. By using the non-local density functional theory (NLDFT) model, the pore sizes were derived from the sorption curve.

\section{Isosteric heat of adsorption.}

The binding energy of $\mathrm{CO}_{2}$ is reflected in the isosteric heat of adsorption, $\mathrm{Q}_{\mathrm{st}}$, defined as

$$
Q_{s t}=R T^{2}\left(\frac{\partial \ln p}{\partial T}\right)_{q}
$$

The calculations are based on the use of the Clausius-Clapeyron equation.

\section{Proton conductivity}


The proton conductivities were investigated by $\mathrm{AC}$ impedance measurements at frequencies from $1 \mathrm{MHz}$ to $1 \mathrm{~Hz}$ with an amplitude of $10 \mathrm{mV}$ at a controlled temperature and relative humidity $(\mathrm{RH})$. The $\mathrm{AC}$ impedance measurements of these films were performance using a SmartLab Electrochemical Workstation. The relative humidities and temperatures were controlled in an Espec SH-221 bench-top temperature and humidity chamber. 70 to 80 $\mathrm{mg}$ of COF samples were pressed into a die under 1000 psi for 5 minutes to obtain uniform pellets (3 $\mathrm{mm}$ in thickness, $1.0 \mathrm{~cm}$ in diameter). The pellet was loaded into custom 2electeode cell between two gold foils to enhance contact, which gave rise to an electrode area of $0.3 \mathrm{~cm}^{2}$, and was then inserted in a humidity chamber maintained at $90 \% \mathrm{RH}$. The conductivity was subsequently measured at different temperatures. When changing to a new temperature, the sample was allowed to equilibrate for $24 \mathrm{~h}$. Proton conductivity was calculated by the following equation:

$$
\sigma=\mathrm{L} /(\mathrm{RA})
$$

where $\sigma$ is proton conductivity $\left(\mathrm{S} \mathrm{cm}^{-1}\right), \mathrm{L}$ is thickness of the pellet $(\mathrm{cm}), \mathrm{A}$ is area of the electrodes $\left(\mathrm{cm}^{2}\right)$, and $\mathrm{R}$ is the resistance $(\Omega)$ of the pellet corresponding to the real $\mathrm{Z}^{\prime}$ Nyquist plot.

The calculated proton conductivity values at different temperatures were fitted to Arrhenius equation shown below to obtain the activation energy Ea for proton conduction process.

$$
\ln (\sigma \mathrm{T})=\ln (\mathrm{A})-\frac{-\mathrm{E}_{\mathrm{a}}}{\mathrm{K}} \frac{1}{\mathrm{~T}}
$$

In this equitation, $\sigma$ is the proton conductivity measured at temperature $\mathrm{T}, \mathrm{A}$ is a constant, and $\mathrm{K}$ is the Boltzmann constant. 


\section{Supporting Tables}

Table S1. Crystallographic data and structural refinement summary for 1 .

\begin{tabular}{|c|c|}
\hline & 1 \\
\hline crystal system & monoclinic \\
\hline space group & $P 2_{1} / \mathrm{c}(14 \#)$ \\
\hline empirical formula & $\mathrm{C}_{24} \mathrm{H}_{12} \mathrm{~N}_{2} \mathrm{O}_{2}$ \\
\hline molecular weight & 360.36 \\
\hline$a(\AA)$ & $9.8013(2)$ \\
\hline$b(\AA)$ & $4.57330(10)$ \\
\hline$c(\AA)$ & $18.4116(6)$ \\
\hline$\alpha\left(^{\circ}\right)$ & 90 \\
\hline$\beta\left(\left(^{\circ}\right)\right.$ & $91.760(2)$ \\
\hline$\gamma\left({ }^{\circ}\right)$ & 90 \\
\hline$V\left(\AA^{3}\right)$ & $824.90(4)$ \\
\hline$Z, d_{\text {calcd }}\left(\mathrm{g} \mathrm{cm}^{-3}\right)$ & $2,1.451$ \\
\hline diffractometer & $\mathrm{CCD}$ \\
\hline$\mu\left(\mathrm{cm}^{-1}\right)$ & 0.756 \\
\hline radiation type & Mo $K \alpha$ \\
\hline $\begin{array}{c}\text { radiation wavelength } \\
(\AA)\end{array}$ & 0.71073 \\
\hline$F(000)$ & 372 \\
\hline goodness of fit & 1.021 \\
\hline temperature $(\mathrm{K})$ & $99.9(5)$ \\
\hline $\begin{array}{l}\text { number of reflections } \\
\text { collected/unique }\end{array}$ & $1390 / 1531$ \\
\hline$R_{\text {int }}$ & 0.0205 \\
\hline$R_{1}(I>2.00 \sigma(I))^{[\mathrm{a}]}$ & 0.0402 \\
\hline$w R_{2}(I>2.00 \sigma(I))^{[\mathrm{b}]}$ & 0.1068 \\
\hline CCDC number & 2005691 \\
\hline
\end{tabular}


Table S2. Screening of conditions for COF synthesis.

\begin{tabular}{|c|c|c|c|c|c|c|}
\hline $\begin{array}{c}\text { Reactant } \\
1(16.6 \\
\text { mg) }\end{array}$ & $\begin{array}{c}\text { Reactant } \\
2(13.0 \\
\text { mg) }\end{array}$ & $\begin{array}{c}\text { Base (4 } \\
\text { eq.) }\end{array}$ & $\begin{array}{l}\text { Solvent (2 } \\
\text { mL) }\end{array}$ & $\begin{array}{c}\text { Temp. } \\
\left({ }^{\circ} \mathrm{C}\right)\end{array}$ & $\begin{array}{c}\text { Time and } \\
\text { atmosphere }\end{array}$ & Result and yield \\
\hline DHPA & BTTA & $\mathrm{K}_{2} \mathrm{CO}_{3}$ & DMF & 105 & $6 \mathrm{~d}, \mathrm{O}_{2}$ & amorphous \\
\hline DHPA & BTTA & $\mathrm{NaOH}$ & DMF & 105 & $6 \mathrm{~d}, \mathrm{O}_{2}$ & crystalline, $31 \%$ \\
\hline DHPA & BTTA & tBuOK & $\mathrm{DMF}$ & 90 & $6 \mathrm{~d}, \mathrm{O}_{2}$ & nearly amorphous \\
\hline DHPA & BTTA & $\mathrm{tBuOK}$ & $\begin{array}{c}\text { DMF/mesity } \\
\text { lene }=1: 1\end{array}$ & 105 & $6 \mathrm{~d}, \mathrm{O}_{2}$ & $\begin{array}{c}\text { little solid } \\
\text { product }\end{array}$ \\
\hline DHPA & BTTA & $\mathrm{tBuOK}$ & DMF & 105 & $3 \mathrm{~d}, \mathrm{O}_{2}$ & crystalline, $48 \%$ \\
\hline DHPA & BTTA & $\mathrm{tBuOK}$ & DMF & 105 & $6 \mathrm{~d}$, vacuum & $\begin{array}{c}\text { impure products } \\
\text { with strange } \\
\text { PXRD }\end{array}$ \\
\hline DHPA & BTTA & $\mathrm{tBuOK}$ & $\mathrm{DMF}$ & 105 & $6 \mathrm{~d}, \mathrm{O}_{2}$ & crystalline, $88 \%$ \\
\hline $\begin{array}{c}\text { Reactant } \\
1(15.8 \\
\mathrm{mg})\end{array}$ & $\begin{array}{c}\text { Reactant } \\
2(17.8 \\
\mathrm{mg})\end{array}$ & $\begin{array}{c}\text { Base (4 } \\
\text { eq.) }\end{array}$ & $\begin{array}{l}\text { Solvent (2 } \\
\text { mL) }\end{array}$ & $\begin{array}{l}\text { Temp. } \\
\left({ }^{\circ} \mathrm{C}\right)\end{array}$ & $\begin{array}{c}\text { Time and } \\
\text { atmosphere }\end{array}$ & Result and yield \\
\hline THTA & PDA & $\mathrm{K}_{2} \mathrm{CO}_{3}$ & DMF & 105 & $6 \mathrm{~d}, \mathrm{O}_{2}$ & amorphous \\
\hline THTA & PDA & $\mathrm{NaOH}$ & $\mathrm{DMF}$ & 105 & $6 \mathrm{~d}, \mathrm{O}_{2}$ & crystalline, $15 \%$ \\
\hline THTA & PDA & $\mathrm{tBuOK}$ & $\mathrm{DMF}$ & 90 & $6 \mathrm{~d}, \mathrm{O}_{2}$ & crystalline, 23 \\
\hline THTA & PDA & $\mathrm{tBuOK}$ & $\begin{array}{c}\text { DMF/mesity } \\
\text { lene }=1: 1\end{array}$ & 105 & $6 \mathrm{~d}, \mathrm{O}_{2}$ & crystalline, $10 \%$ \\
\hline THTA & PDA & $\mathrm{tBuOK}$ & $\mathrm{DMF}$ & 105 & $3 \mathrm{~d}, \mathrm{O}_{2}$ & crystalline, $61 \%$ \\
\hline THTA & PDA & $\mathrm{tBuOK}$ & DMF & 105 & $6 \mathrm{~d}$, vacuum & $\begin{array}{c}\text { impure products } \\
\text { with strange } \\
\text { PXRD }\end{array}$ \\
\hline THTA & PDA & $\mathrm{tBuOK}$ & DMF & 105 & $6 \mathrm{~d}, \mathrm{O}_{2}$ & crystalline, $92 \%$ \\
\hline
\end{tabular}


Table S3. Elemental analysis results of GS-COF-1 and -2 .

\begin{tabular}{|c|c|c|c|c|}
\hline COF & & $\mathrm{C} \%$ & $\mathrm{H} \%$ & $\mathrm{~N} \%$ \\
\hline \multirow{2}{*}{ GS-COF-1 } & Calcd. & 75.00 & 1.57 & 10.93 \\
\cline { 2 - 5 } & Found & 72.86 & 1.84 & 9.99 \\
\hline \multirow{2}{*}{ GS-COF-2 } & Calcd. & 75.00 & 1.57 & 10.93 \\
\cline { 2 - 5 } & Found & 76.55 & 1.81 & 10.02 \\
\hline
\end{tabular}


Table S4. Lattice parameters of GS-COF-1 and -2 .

\begin{tabular}{|c|c|c|c|c|}
\hline & \multicolumn{2}{|c|}{ GS-COF-1 } & \multicolumn{2}{|c|}{ GS-COF-2 } \\
\hline & $\begin{array}{l}\text { Eclipsed AA } \\
\text { mode }\end{array}$ & $\begin{array}{l}\text { Staggered AB } \\
\text { mode }\end{array}$ & $\begin{array}{c}\text { Eclipsed AA } \\
\text { mode }\end{array}$ & $\begin{array}{l}\text { Staggered AB } \\
\text { mode }\end{array}$ \\
\hline Formula & $\mathrm{C}_{48} \mathrm{H}_{12} \mathrm{O}_{6} \mathrm{~N}_{6}$ & $\mathrm{C}_{48} \mathrm{H}_{12} \mathrm{O}_{6} \mathrm{~N}_{6}$ & $\mathrm{C}_{48} \mathrm{H}_{12} \mathrm{O}_{6} \mathrm{~N}_{6}$ & $\mathrm{C}_{48} \mathrm{H}_{12} \mathrm{O}_{6} \mathrm{~N}_{6}$ \\
\hline $\begin{array}{l}\text { Formula } \\
\text { weight }\end{array}$ & 768.66 & 768.66 & 768.66 & 768.66 \\
\hline $\begin{array}{l}\text { Crystal } \\
\text { system }\end{array}$ & trigonal & hexagonal & hexagonal & hexagonal \\
\hline Space group & P6/M (No.175) & $\mathrm{P} 6_{3} / \mathrm{M}(\mathrm{No} .176)$ & P6/M (No.175) & $\mathrm{P} 6_{3} / \mathrm{M}(\mathrm{No} .176)$ \\
\hline $\begin{array}{l}\text { Unit cell } \\
\text { dimensions }\end{array}$ & $\begin{array}{c}a=b=21.2315 \\
\AA, c=3.6125 \AA \\
\alpha=\beta=90^{\circ}, \gamma= \\
120^{\circ}\end{array}$ & $\begin{array}{c}a=b=21.2268 \\
\AA, c=6.5521 \AA \\
\alpha=\beta=90^{\circ}, \gamma= \\
120^{\circ}\end{array}$ & $\begin{array}{c}a=b=21.3752 \\
\AA, c=3.612 \AA \\
\alpha=\beta=90^{\circ}, \gamma= \\
120^{\circ}\end{array}$ & $\begin{array}{c}a=b=21.3688 \\
\AA, c=6.6032 \AA \\
\alpha=\beta=90^{\circ}, \gamma= \\
120^{\circ}\end{array}$ \\
\hline Cell volume & $1410.76 \AA^{3}$ & $2561.38 \AA^{3}$ & $1428.34 \AA^{3}$ & $2611.59 \AA^{3}$ \\
\hline Void space & $\begin{array}{c}644.72 \AA^{3} \\
(45.7 \% \text { of unit } \\
\text { cell) }\end{array}$ & $\begin{array}{c}632.66 \AA^{3} \\
(24.7 \% \text { of unit } \\
\text { cell) }\end{array}$ & $\begin{array}{c}661.32 \AA^{3} \\
(46.3 \% \text { of unit } \\
\text { cell) }\end{array}$ & $\begin{array}{c}739.08 \AA^{3} \\
(28.3 \% \text { of unit } \\
\text { cell) }\end{array}$ \\
\hline
\end{tabular}


Table S5. Atomistic coordinates for the AA-stacking mode of GS-COF-1 optimized by using DFT method in CASTEP with PBE as functional (Space group: $P-3 ; a=b=21.2315 \AA, c=$ $3.6125 \AA ; \alpha=\beta=90^{\circ}, \gamma=120^{\circ}$ ).

\begin{tabular}{|c|c|c|c|}
\hline Atom & $\boldsymbol{x} / \boldsymbol{a}$ & $\boldsymbol{y} / \boldsymbol{b}$ & $\boldsymbol{z} / \boldsymbol{c}$ \\
\hline $\mathrm{C}$ & 0.6977 & 0.40926 & 0.5 \\
\hline $\mathrm{C}$ & 0.62168 & 0.36442 & 0.5 \\
\hline $\mathrm{C}$ & 0.57589 & 0.39727 & 0.5 \\
\hline $\mathrm{O}$ & 0.61126 & 0.47191 & 0.5 \\
\hline $\mathrm{C}$ & 0.55925 & 0.49244 & 0.5 \\
\hline $\mathrm{C}$ & 0.48882 & 0.4299 & 0.5 \\
\hline $\mathrm{C}$ & 0.5011 & 0.36893 & 0.5 \\
\hline $\mathrm{C}$ & 0.57375 & 0.56366 & 0.5 \\
\hline $\mathrm{C}$ & 0.44645 & 0.29492 & 0.5 \\
\hline $\mathrm{N}$ & 0.40103 & 0.23398 & 0.5 \\
\hline $\mathrm{H}$ & 0.72111 & 0.46798 & 0.5 \\
\hline $\mathrm{H}$ & 0.62876 & 0.61018 & 0.5 \\
\hline
\end{tabular}


Table S6. Atomistic coordinates for the AB-stacking mode of GS-COF-1 optimized by using DFT method in CASTEP with PBE as functional (Space group: $P 6_{3} / M ; a=b=21.2268 \AA, c$ $=6.5521 \AA ; \alpha=\beta=90^{\circ}, \gamma=120^{\circ}$ ).

\begin{tabular}{|c|c|c|c|}
\hline Atom & $x / a$ & $y / b$ & $z / c$ \\
\hline $\mathrm{C}$ & 1.63538 & 1.25732 & 0.25 \\
\hline $\mathrm{C}$ & 1.71149 & 1.30198 & 0.25 \\
\hline $\mathrm{C}$ & 1.75714 & 1.26894 & 0.25 \\
\hline $\mathrm{O}$ & 1.72163 & 1.19429 & 0.25 \\
\hline $\mathrm{C}$ & 1.77345 & 1.17352 & 0.25 \\
\hline $\mathrm{C}$ & 1.84397 & 1.23598 & 0.25 \\
\hline $\mathrm{C}$ & 1.83195 & 1.29712 & 0.25 \\
\hline $\mathrm{C}$ & 1.75898 & 1.10226 & 0.25 \\
\hline $\mathrm{C}$ & 1.88756 & 1.37079 & 0.25 \\
\hline $\mathrm{N}$ & 1.935 & 1.43092 & 0.25 \\
\hline $\mathrm{H}$ & 1.6118 & 1.19855 & 0.25 \\
\hline $\mathrm{H}$ & 1.70394 & 1.05577 & 0.25 \\
\hline $\mathrm{C}$ & 2.03075 & 1.07583 & 0.25 \\
\hline $\mathrm{C}$ & 1.95482 & 1.03081 & 0.25 \\
\hline $\mathrm{C}$ & 1.90902 & 1.06342 & 0.25 \\
\hline $\mathrm{O}$ & 1.9441 & 1.13798 & 0.25 \\
\hline $\mathrm{C}$ & 1.89194 & 1.15835 & 0.25 \\
\hline $\mathrm{C}$ & 1.82158 & 1.09582 & 0.25 \\
\hline $\mathrm{C}$ & 1.8341 & 1.03499 & 0.25 \\
\hline $\mathrm{C}$ & 1.90643 & 1.2295 & 0.25 \\
\hline $\mathrm{C}$ & 1.7797 & 0.96098 & 0.25 \\
\hline $\mathrm{N}$ & 1.73389 & 0.90009 & 0.25 \\
\hline $\mathrm{H}$ & 2.05381 & 1.13447 & 0.25 \\
\hline $\mathrm{H}$ & 1.96136 & 1.27613 & 0.25 \\
\hline
\end{tabular}


Table S7. Atomistic coordinates for the AA-stacking mode of GS-COF-2 optimized by using DFT method in CASTEP with PBE as functional (Space group: P6/M; $a=b=21.3752 \AA, c=$ $3.612 \AA ; \alpha=\beta=90^{\circ}, \gamma=120^{\circ}$ ).

\begin{tabular}{|c|c|c|c|}
\hline Atom & $\boldsymbol{x} / \boldsymbol{a}$ & $\boldsymbol{y} / \boldsymbol{b}$ & $\boldsymbol{z} / \boldsymbol{c}$ \\
\hline $\mathrm{C}$ & 0.59565 & 0.27221 & 0.5 \\
\hline $\mathrm{C}$ & 0.65713 & 0.26445 & 0.5 \\
\hline $\mathrm{C}$ & 0.53479 & 0.19984 & 0.5 \\
\hline $\mathrm{C}$ & 0.56287 & 0.1534 & 0.5 \\
\hline $\mathrm{O}$ & 0.63738 & 0.1931 & 0.5 \\
\hline $\mathrm{C}$ & 0.72024 & 0.53835 & 0.5 \\
\hline $\mathrm{N}$ & 0.76724 & 0.59806 & 0.5 \\
\hline $\mathrm{C}$ & 0.45504 & 0.53078 & 0.5 \\
\hline $\mathrm{C}$ & 0.42479 & 0.45501 & 0.5 \\
\hline $\mathrm{C}$ & 0.46898 & 0.4248 & 0.5 \\
\hline $\mathrm{H}$ & 0.36645 & 0.41936 & 0.5 \\
\hline $\mathrm{H}$ & 0.44462 & 0.36625 & 0.5 \\
\hline
\end{tabular}


Table S8. Atomistic coordinates for the AB-stacking mode of GS-COF-2 optimized by using DFT method in CASTEP with PBE as functional (Space group: $P 6_{3} / M ; a=b=21.3688 \AA, c$ $=6.6032 \AA ; \alpha=\beta=90^{\circ}, \gamma=120^{\circ}$ ).

\begin{tabular}{|c|c|c|c|}
\hline Atom & $x / a$ & $y / b$ & $z / c$ \\
\hline $\mathrm{C}$ & 0.92888 & -0.06088 & 0.25 \\
\hline $\mathrm{C}$ & 0.99015 & -0.06896 & 0.25 \\
\hline $\mathrm{C}$ & 0.86783 & -0.13316 & 0.25 \\
\hline $\mathrm{C}$ & 0.89558 & -0.18003 & 0.25 \\
\hline $\mathrm{O}$ & 0.9701 & -0.1404 & 0.25 \\
\hline $\mathrm{C}$ & 0.05464 & -0.79496 & 0.25 \\
\hline $\mathrm{N}$ & 0.10251 & -0.73559 & 0.25 \\
\hline $\mathrm{C}$ & 0.78897 & -0.8021 & 0.25 \\
\hline $\mathrm{C}$ & 0.75878 & -0.87788 & 0.25 \\
\hline $\mathrm{C}$ & 0.80304 & -0.90796 & 0.25 \\
\hline $\mathrm{H}$ & 0.70045 & -0.91344 & 0.25 \\
\hline $\mathrm{H}$ & 0.7788 & -0.9665 & 0.25 \\
\hline $\mathrm{C}$ & 0.73774 & -0.6054 & 0.25 \\
\hline $\mathrm{C}$ & 0.67613 & -0.59775 & 0.25 \\
\hline $\mathrm{C}$ & 0.79852 & -0.5329 & 0.25 \\
\hline $\mathrm{C}$ & 0.77017 & -0.4866 & 0.25 \\
\hline $\mathrm{O}$ & 0.69574 & -0.52644 & 0.25 \\
\hline $\mathrm{C}$ & 0.61386 & -0.87193 & 0.25 \\
\hline $\mathrm{N}$ & 0.56786 & -0.93215 & 0.25 \\
\hline $\mathrm{C}$ & 0.87897 & -0.8635 & 0.25 \\
\hline $\mathrm{C}$ & 0.9092 & -0.78778 & 0.25 \\
\hline $\mathrm{C}$ & 0.86498 & -0.75754 & 0.25 \\
\hline $\mathrm{H}$ & 0.96757 & -0.75219 & 0.25 \\
\hline $\mathrm{H}$ & 0.88915 & -0.69899 & 0.25 \\
\hline
\end{tabular}


Table S9. Proton conductivity of COF materials reported to date.

\begin{tabular}{|c|c|c|c|c|c|}
\hline COFs & $\begin{array}{l}\text { Type of the } \\
\text { linkages }\end{array}$ & $\begin{array}{c}\sigma \\
\left(\mathrm{S} \mathrm{cm^{-1 }}\right)\end{array}$ & $\begin{array}{c}\text { Ea } \\
(\mathrm{eV})\end{array}$ & Condition & Ref \\
\hline $\begin{array}{l}\mathrm{H}_{3} \mathrm{PO}_{4} @ \text { TPB- } \\
\text { DMeTP-COF }\end{array}$ & imine & $1.91 \times 10^{-1}$ & 0.34 & $\begin{array}{c}433 \mathrm{~K}, \\
\text { anhydrous }\end{array}$ & S8 \\
\hline NKCOF & imine & $1.13 \times 10^{-1}$ & 0.14 & $\begin{array}{c}353 \mathrm{~K}, 98 \% \\
\mathrm{RH}\end{array}$ & S9 \\
\hline PTSA@TpAzo & $\beta$-ketoenamine & $7.8 \times 10^{-2}$ & 0.11 & $\begin{array}{c}353 \mathrm{~K}, 95 \% \\
\mathrm{RH}\end{array}$ & S10 \\
\hline NUS-10(R) & $\beta$-ketoenamine & $3.96 \times 10^{-2}$ & not reported & $\begin{array}{c}298 \mathrm{~K}, 97 \% \\
\text { RH }\end{array}$ & $\mathrm{S} 11$ \\
\hline BIP & imidazole & $3.2 \times 10^{-2}$ & 0.31 & $\begin{array}{c}368 \mathrm{~K}, 95 \% \\
\mathrm{RH}\end{array}$ & S12 \\
\hline aza-COF-2 & $\begin{array}{l}\text { aromatic } \\
\text { pyrazine }\end{array}$ & $4.8 \times 10^{-3}$ & 0.45 & $\begin{array}{c}323 \mathrm{~K}, 97 \% \\
\text { RH }\end{array}$ & $\mathrm{S} 13$ \\
\hline $\begin{array}{c}\text { im@TPB- } \\
\text { DMTP-COF }\end{array}$ & imine & $4.3 \times 10^{-3}$ & 0.38 & $\begin{array}{c}403 \mathrm{~K}, \\
\text { anhydrous }\end{array}$ & S14 \\
\hline EB-COF:PW 12 & $\beta$-ketoenamine & $3.2 \times 10^{-3}$ & 0.24 & $\begin{array}{c}298 \mathrm{~K}, 97 \% \\
\text { RH }\end{array}$ & $\mathrm{S} 15$ \\
\hline $\begin{array}{c}\text { PA@TpBpy- } \\
\text { MC }\end{array}$ & $\beta$-ketoenamine & $2.5 \times 10^{-3}$ & 0.11 & $\begin{array}{c}393 \mathrm{~K}, \\
\text { anhydrous }\end{array}$ & S16 \\
\hline PA@Tp-Azo & $\beta$-ketoenamine & $9.9 \times 10^{-4}$ & 0.11 & $\begin{array}{c}332 \mathrm{~K}, 98 \% \\
\text { RH }\end{array}$ & S17 \\
\hline $\begin{array}{c}\text { Phytic@TpPa- } \\
\text { (SOH-Py) }\end{array}$ & $\beta$-ketoenamine & $5.0 \times 10^{-4}$ & 0.16 & $\begin{array}{c}393 \mathrm{~K}, \\
\text { anhydrous }\end{array}$ & $\mathrm{S} 18$ \\
\hline DBC-2P & imine & $4.5 \times 10^{-5}$ & 0.2 & $\begin{array}{c}343 \mathrm{~K}, 98 \% \\
\mathrm{RH}\end{array}$ & S19 \\
\hline RT-COF-1 & imine & $1.83 \times 10^{-5}$ & not reported & $\begin{array}{c}313 \mathrm{~K}, 100 \% \\
\text { RH }\end{array}$ & $\mathrm{S} 20$ \\
\hline $\begin{array}{c}\text { GS-COF-1- } \\
\text { COOH }\end{array}$ & & $1.21 \times 10^{-3}$ & 0.29 & & \\
\hline $\begin{array}{c}\text { GS-COF-2- } \\
\text { COOH }\end{array}$ & & $1.38 \times 10^{-3}$ & 0.27 & & \\
\hline $\begin{array}{c}\mathrm{H}_{3} \mathrm{PO}_{4} @ \mathrm{GS}- \\
\mathrm{COF}-1- \\
\mathrm{COOH}\end{array}$ & benzofuran & $3.45 \times 10^{-2}$ & 0.23 & $\begin{array}{c}353 \mathrm{~K}, 90 \% \\
\mathrm{RH}\end{array}$ & $\begin{array}{l}\text { This } \\
\text { work }\end{array}$ \\
\hline $\begin{array}{c}\mathrm{H}_{3} \mathrm{PO}_{4} @ G S- \\
\text { COF-2- } \\
\text { COOH }\end{array}$ & & $4.35 \times 10^{-2}$ & 0.21 & & \\
\hline
\end{tabular}




\section{Supporting Figures}

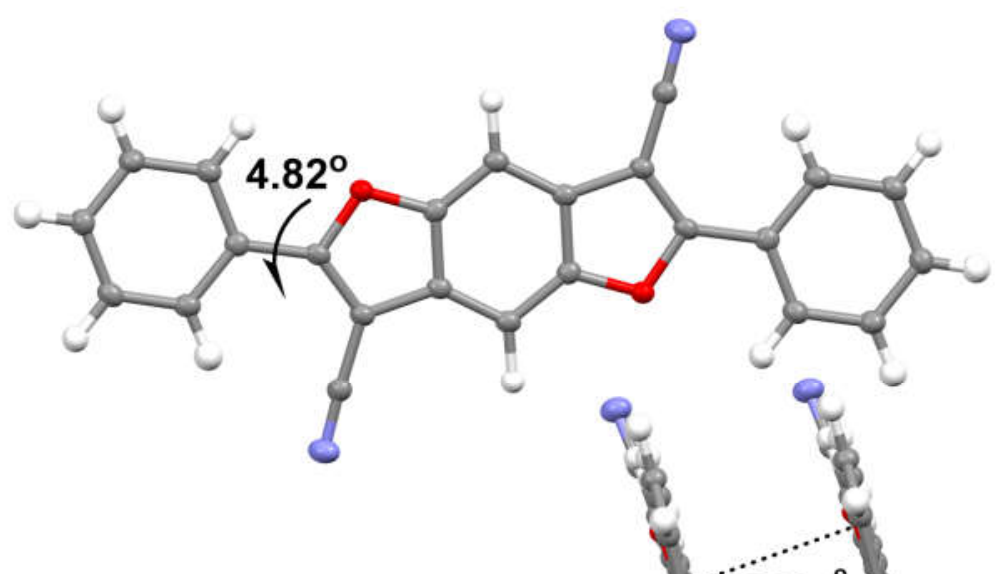

$3.323 \AA$

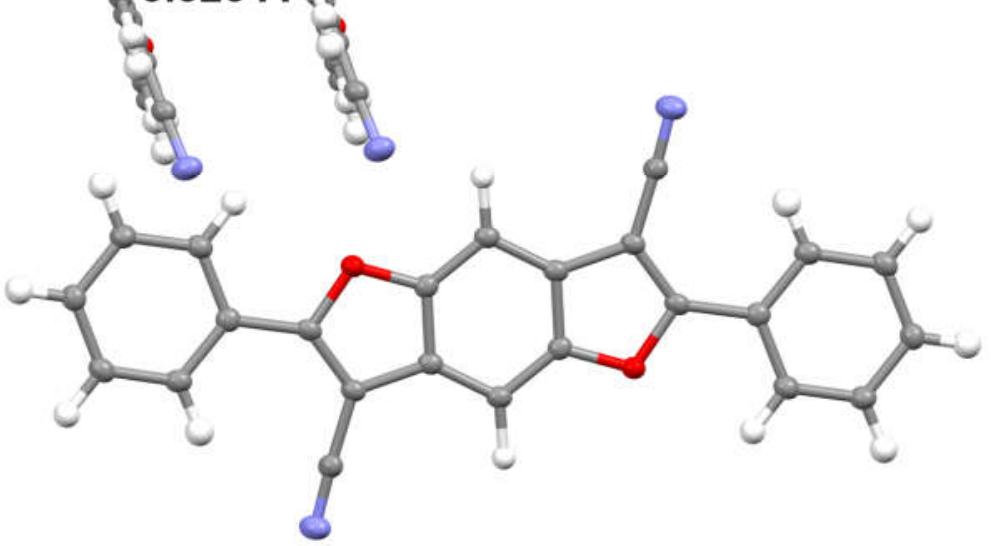

Figure S1. Single-crystal structure of molecular analogue 1. 


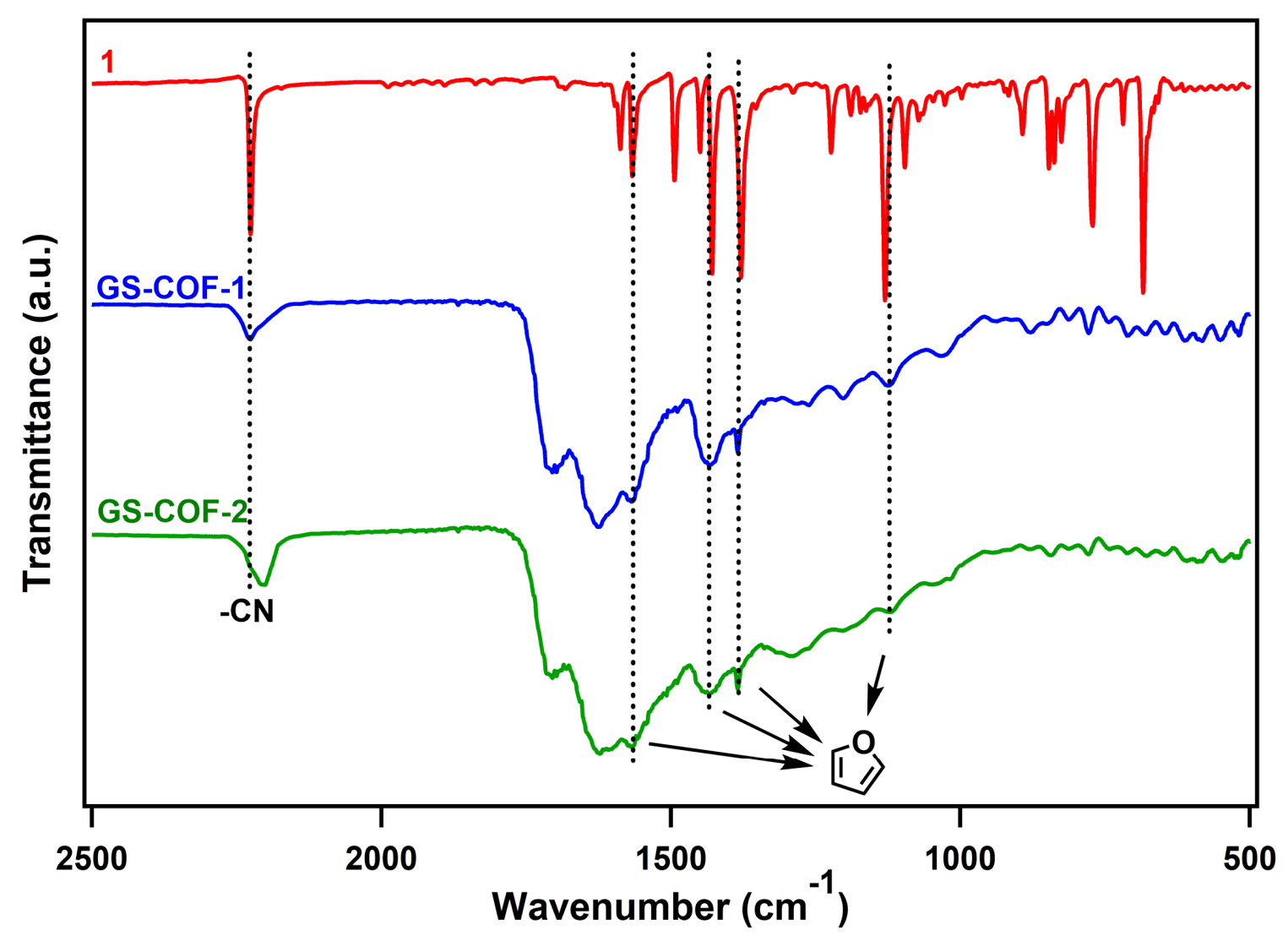

Figure S2. FT-IR spectra of GS-COF-1 and -2 compared with molecular analog 1. 


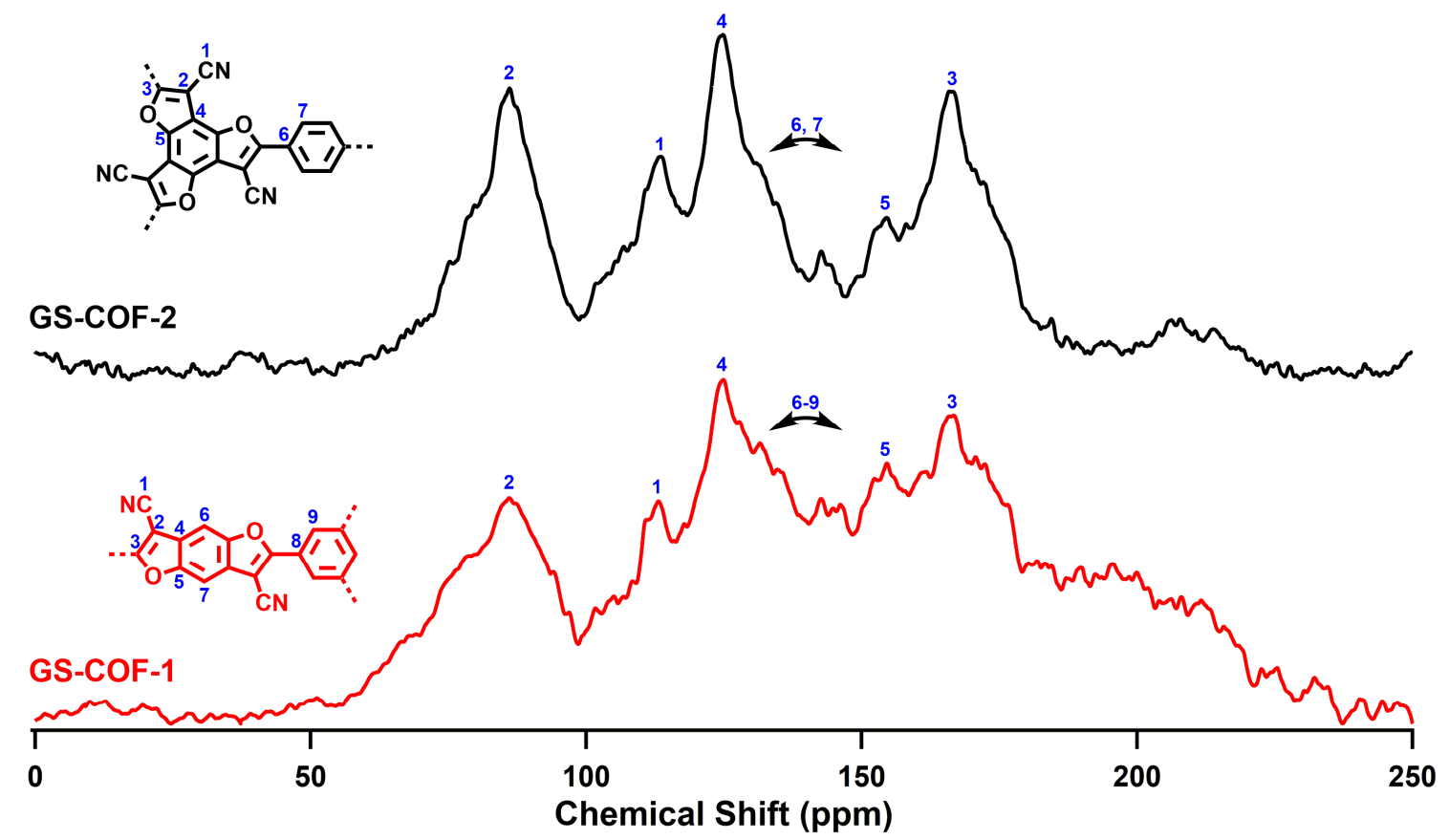

Figure S3. Solid state ${ }^{13} \mathrm{C}$ CP-MAS NMR spectra of GS-COF-1 and -2 . 

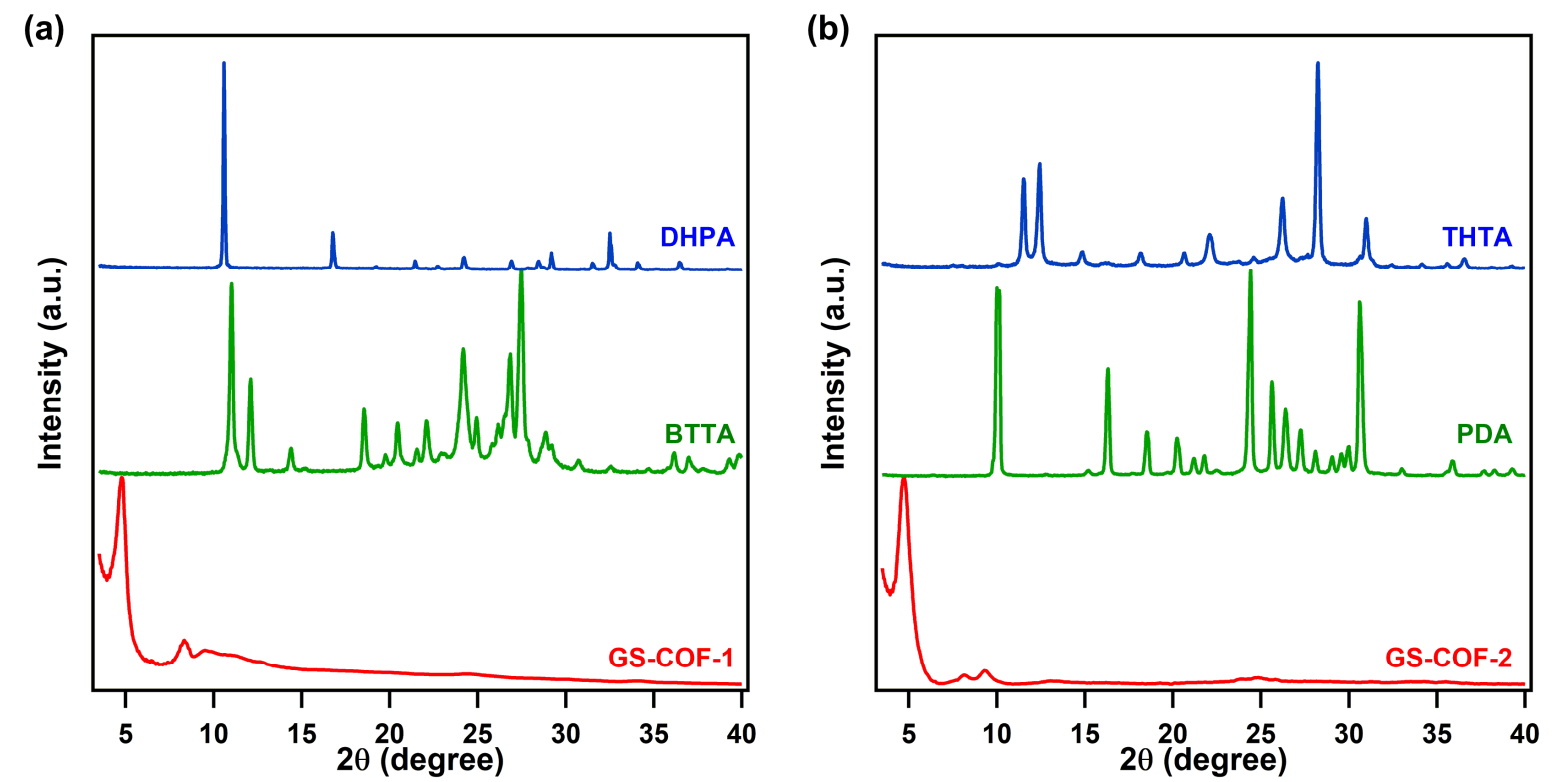

Figure S4. (a) Comparison of PXRD patterns of GS-COF-1 with DHPA and BTTA. (b) Comparison of PXRD patterns of GS-COF-2 with THTA and PDA. 

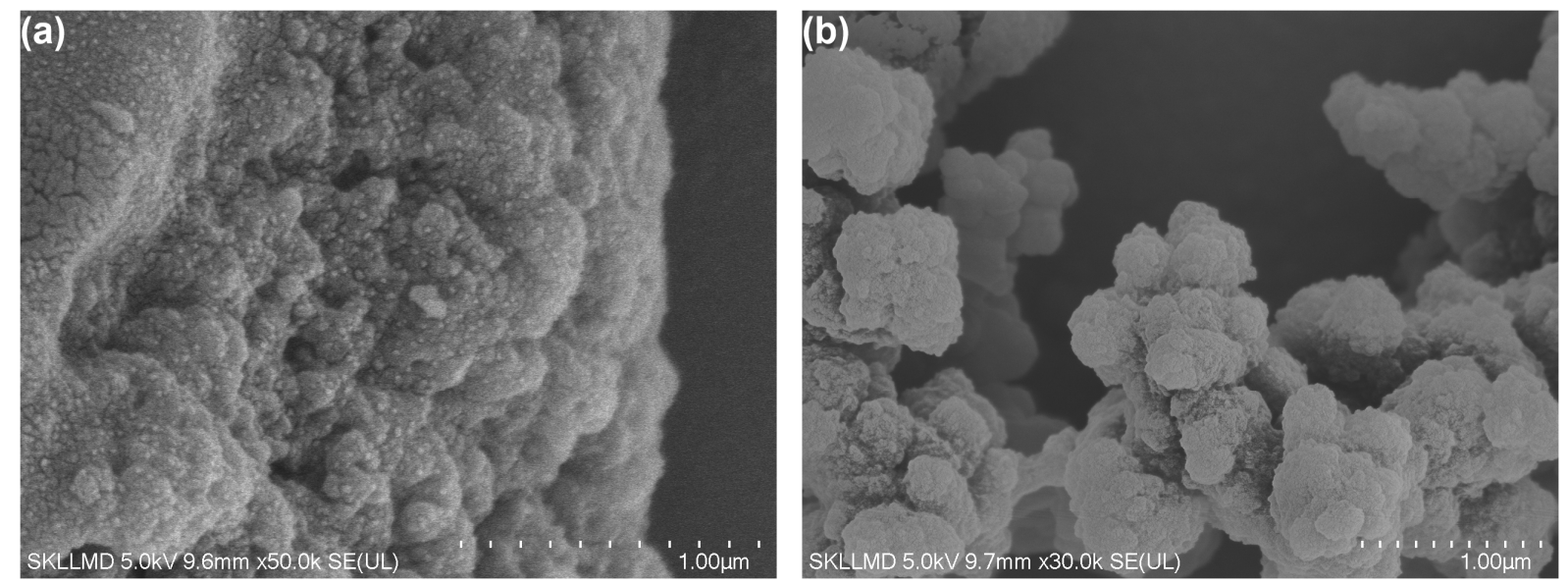

Figure S5. SEM images of (a) GS-COF-1 and (b) GS-COF-2. 
(a)
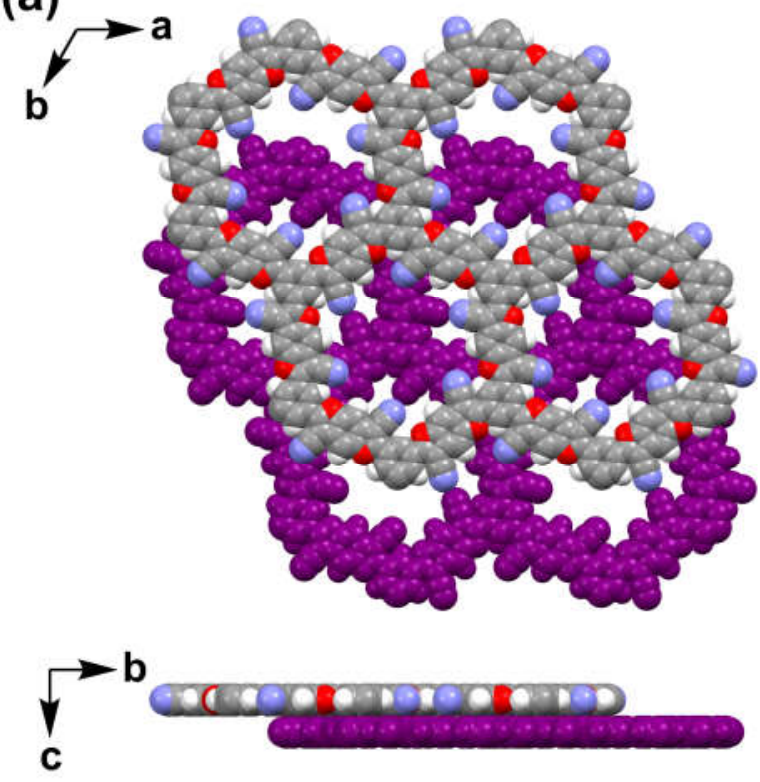

(b)

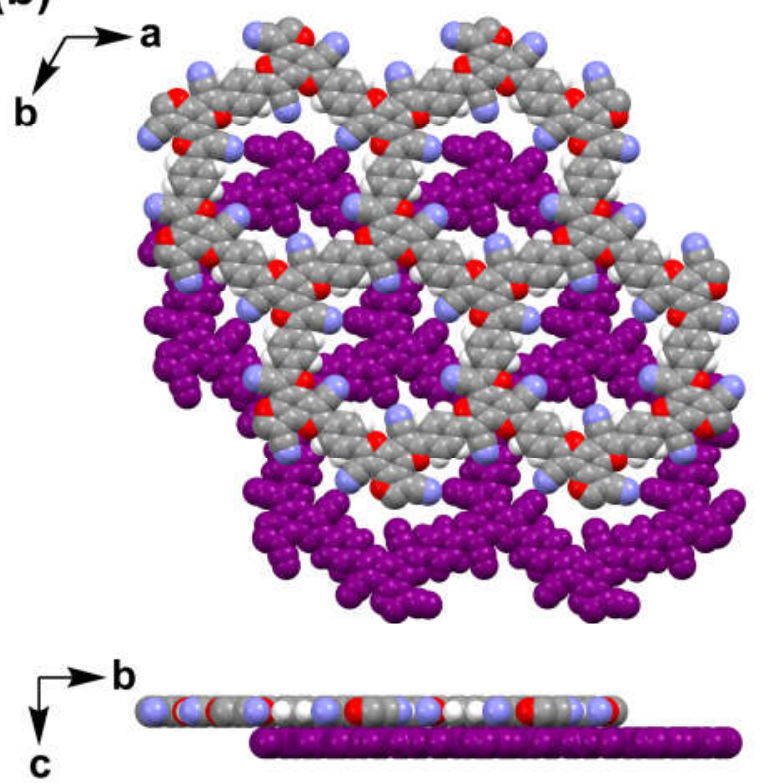

Figure S6. Top and side views of the space-filling models of (a) GS-COF-1 and (b) GS-COF2 in staggered stacking mode. Color code: H, white; C, gray; N; blue; O, red. The lower layers were marked in purple. 
(a)

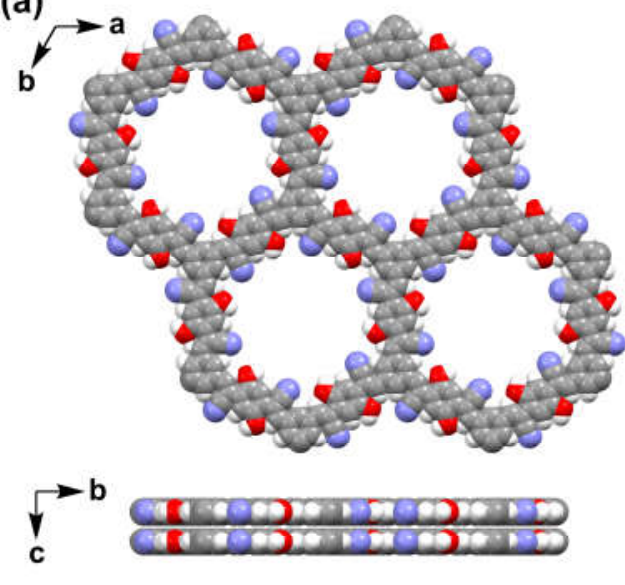

(c)

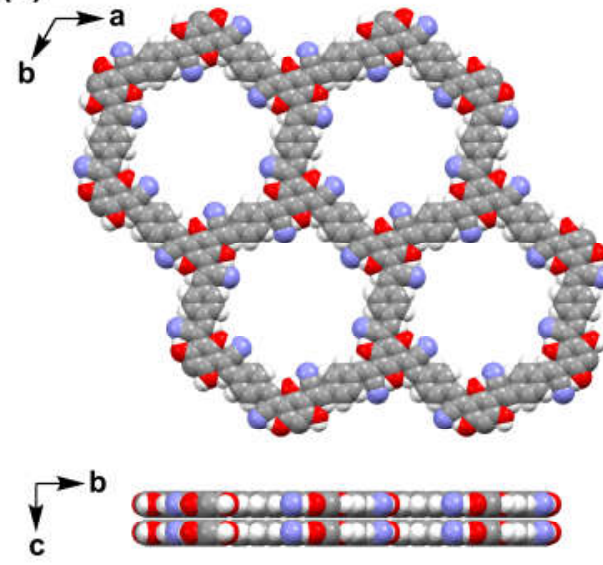

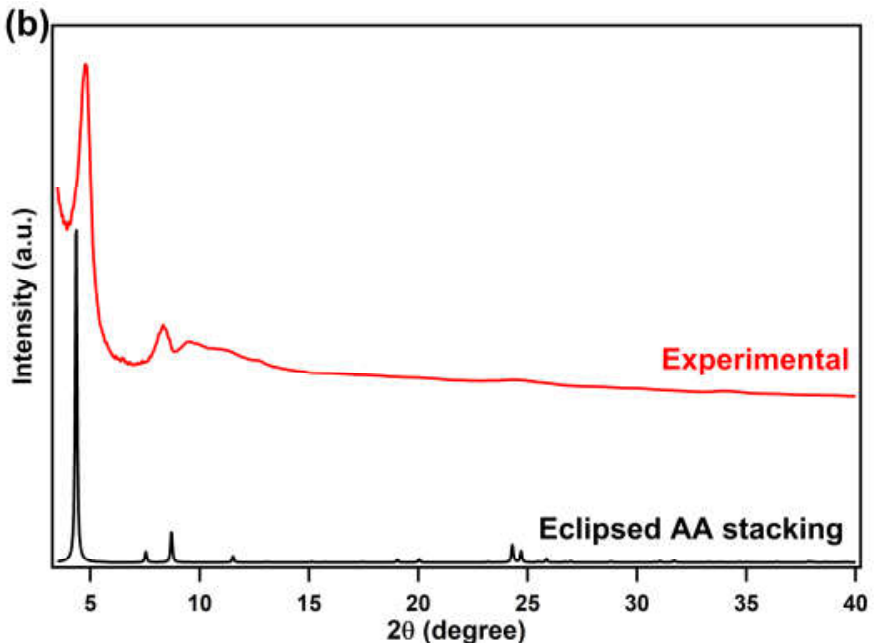

(d)

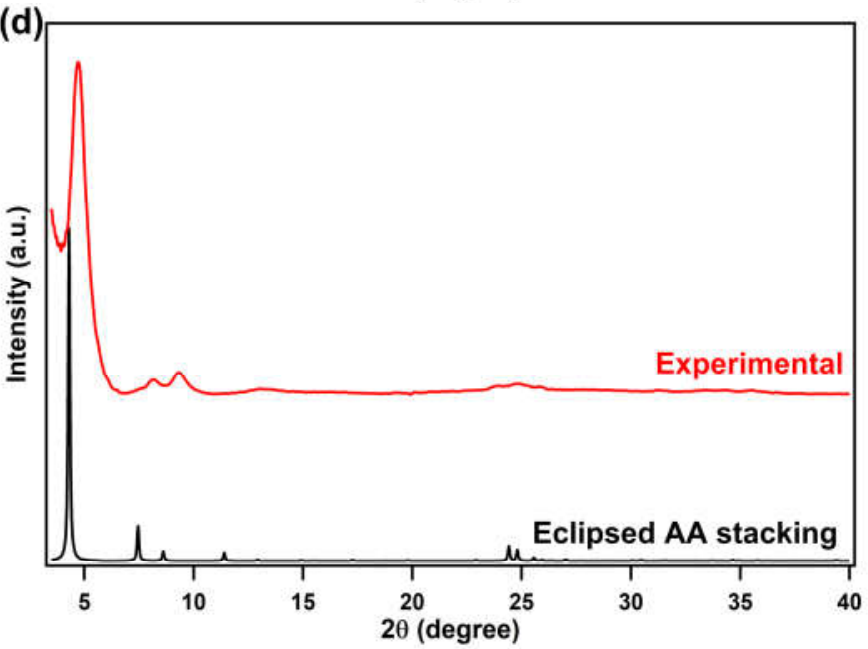

Figure S7. Structural modeling of two imaginary frameworks which were produced by Knoevenagel condensation of the same building blocks but without subsquent cyanide migration, ring-closure and oxidation reactions. (a) and (b) Their structure modelings. (c) and (d) Their calculated PXRD patterns in comparison with the experimental patterns of GS-COF1 and -2. The calculated PXRD patterns can not match with the experimental patterns of GSCOF-1 and -2 , demonstrating the two models were not the COF structures and indicating consecutive irreversibe reactions occurred to yield cyano substituted benzofuran rings. 

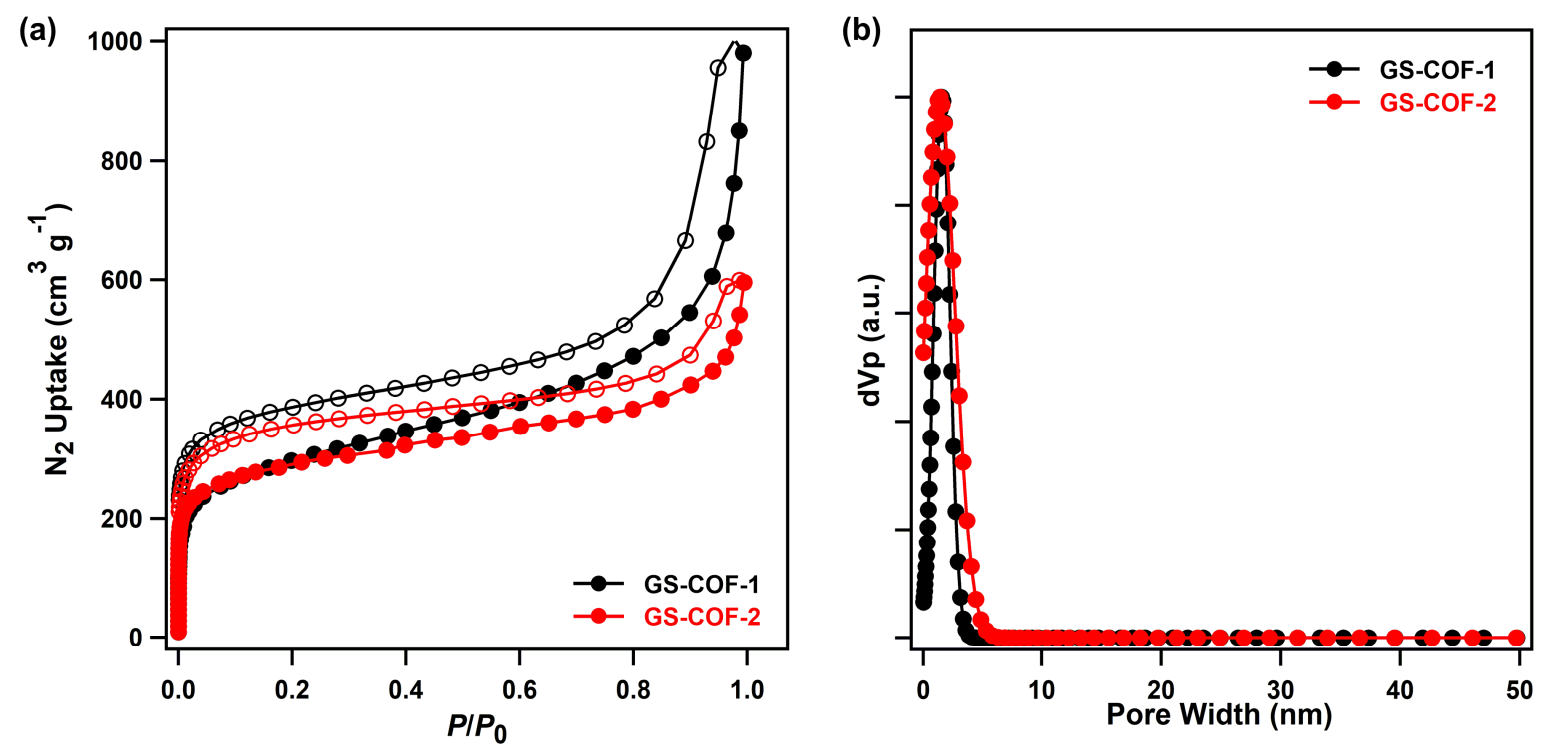

Figure S8. (a) $\mathrm{N}_{2}$ adsorption isotherms of GS-COF-1 and -2 at $77 \mathrm{~K}$. Filled and unfilled circles represent the adsorption and desorption branches, respectively. (b) Pore size distributions of GS-COF-1 and -2 calculated from DFT fitting of the adsorption branch of the $\mathrm{N}_{2}$ adsorption isotherms at $77 \mathrm{~K}$. 


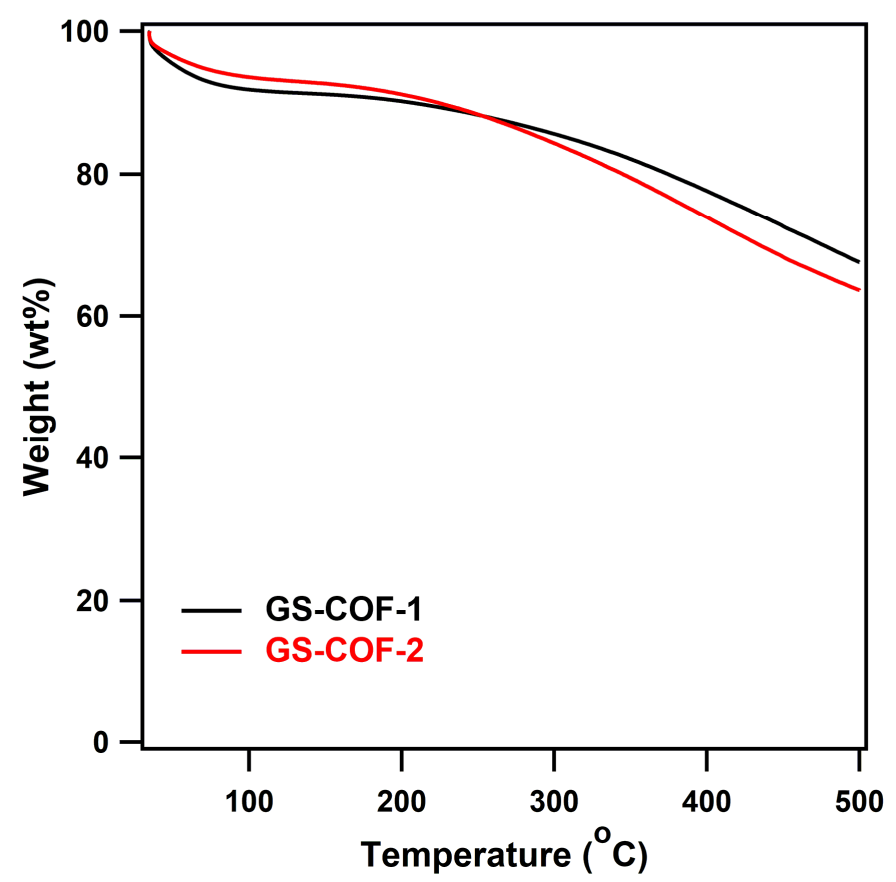

Figure S9. TG curves of GS-COF-1 and -2 .

The two COFs were stable until $200{ }^{\circ} \mathrm{C}$. At $60{ }^{\circ} \mathrm{C}$ the two COFs lost the residual organic solvents (methanol, acetone, THF) in the pores. The weight loss $(\sim 23 \%)$ from 200 to $500{ }^{\circ} \mathrm{C}$ matched well with the proportion of cyano groups $(20.2 \%)$ on the frameworks, indicating the weight loss from 200 to $500{ }^{\circ} \mathrm{C}$ originated from the decomposition of cyano groups on the frameworks. 

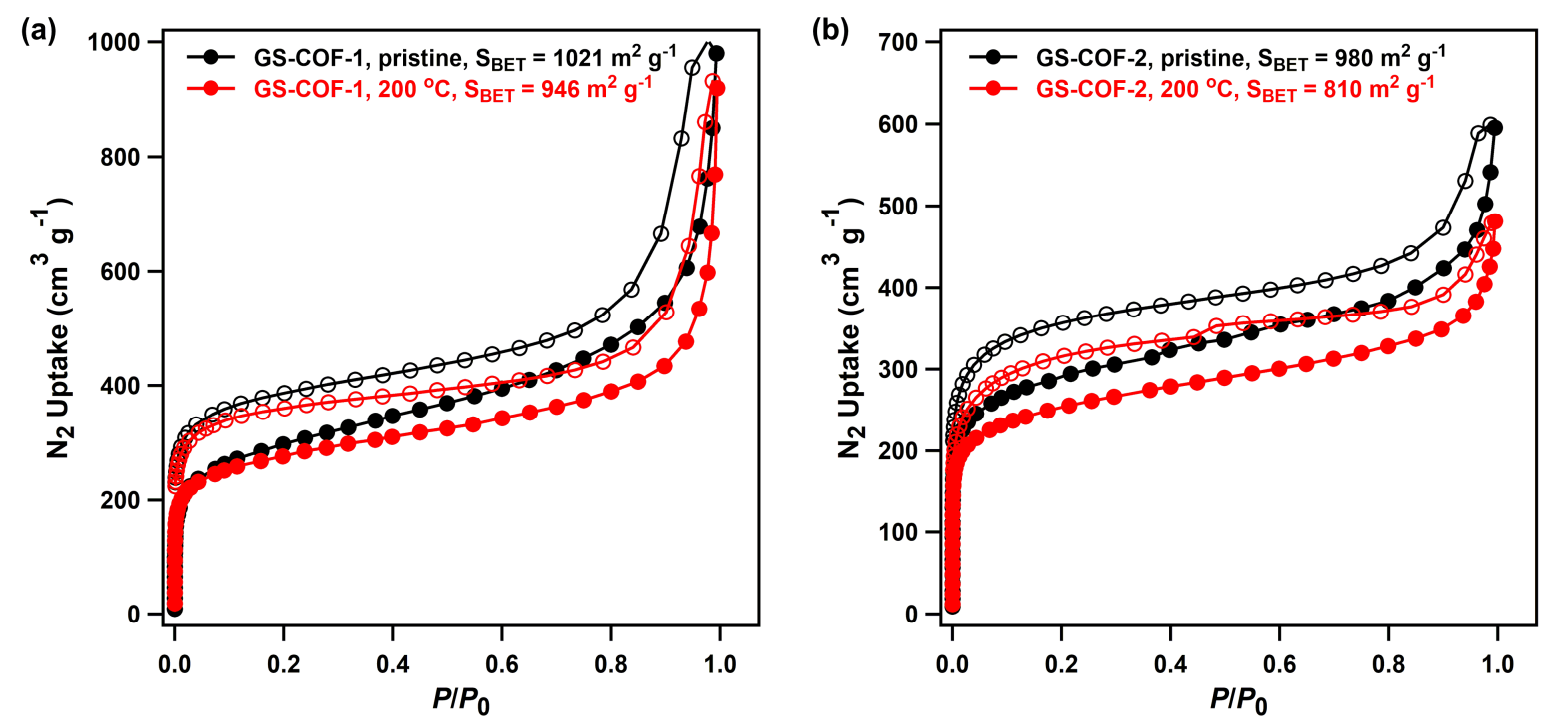

Figure S10. $\mathrm{N}_{2}$ adsorption isotherms of pristine and $200{ }^{\circ} \mathrm{C}$-treated (a) GS-COF-1 and (b) -2 at $77 \mathrm{~K}$.

The two COFs were firstly heated up at $200{ }^{\circ} \mathrm{C}$ for $4 \mathrm{~h}$ under nitrogen, and then were subjected to measure $\mathrm{N}_{2}$ adsorption isotherms. The BET surface areas of GS-COF-1 and -2 after $200{ }^{\circ} \mathrm{C}$ treatment were calculated to be 946 and $810 \mathrm{~m}^{2} \mathrm{~g}^{-1}$, respectively, indicating the two COFs were stable under $200^{\circ} \mathrm{C}$. 

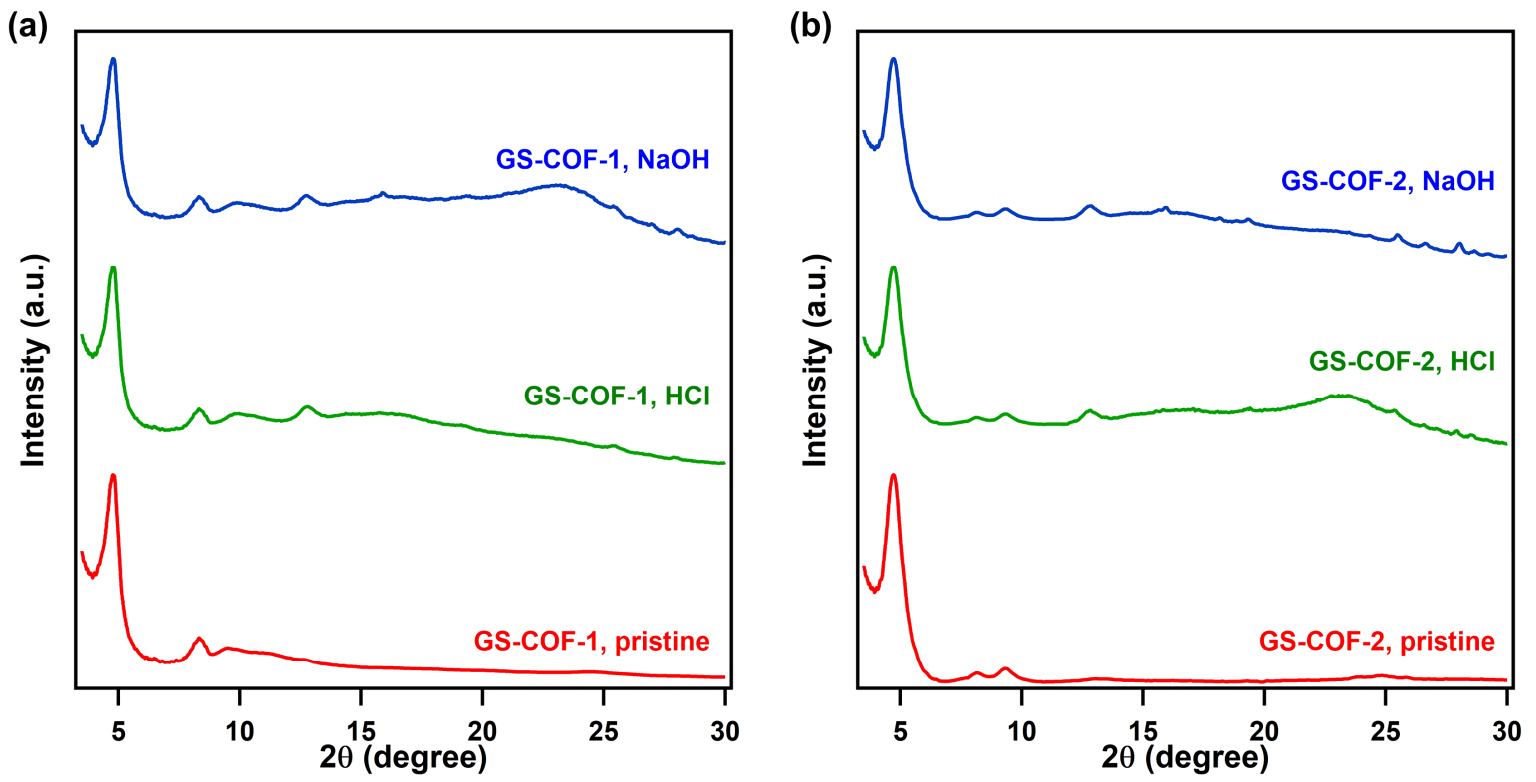

Figure S11. Comparison of PXRD patterns of pristine, $6 \mathrm{M} \mathrm{HCl}$ and $6 \mathrm{M} \mathrm{NaOH}$ treated (a) GS-COF-1 and (b) GS-COF-2 showing the retention of crystallinity after treatment. 

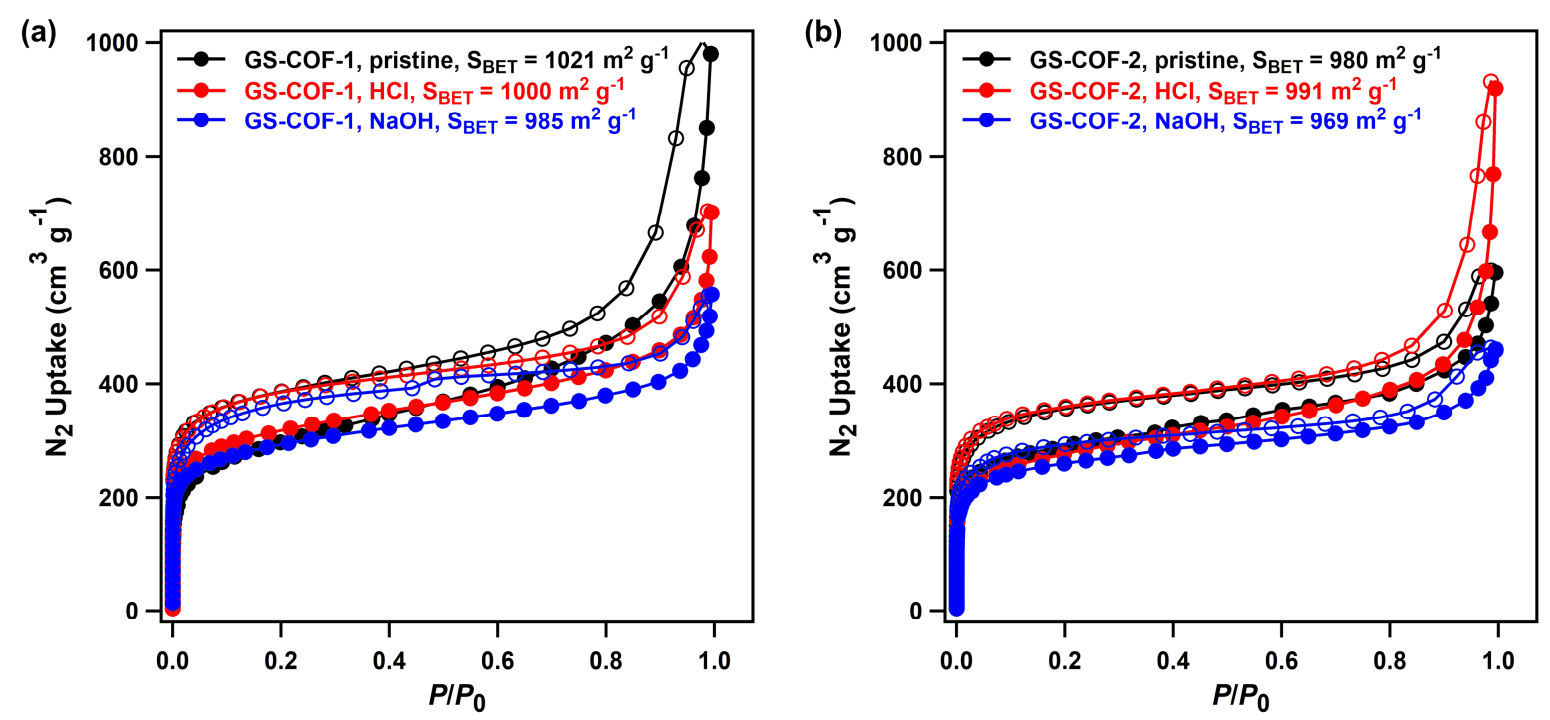

Figure S12. Comparison of $\mathrm{N}_{2}$ sorption isotherms of pristine, $6 \mathrm{M} \mathrm{HCl}$ and $6 \mathrm{M} \mathrm{NaOH}$ treated (a) GS-COF-1 and (b) GS-COF-2. Solid and open circles represent the adsorption and desorption branches, respectively. 

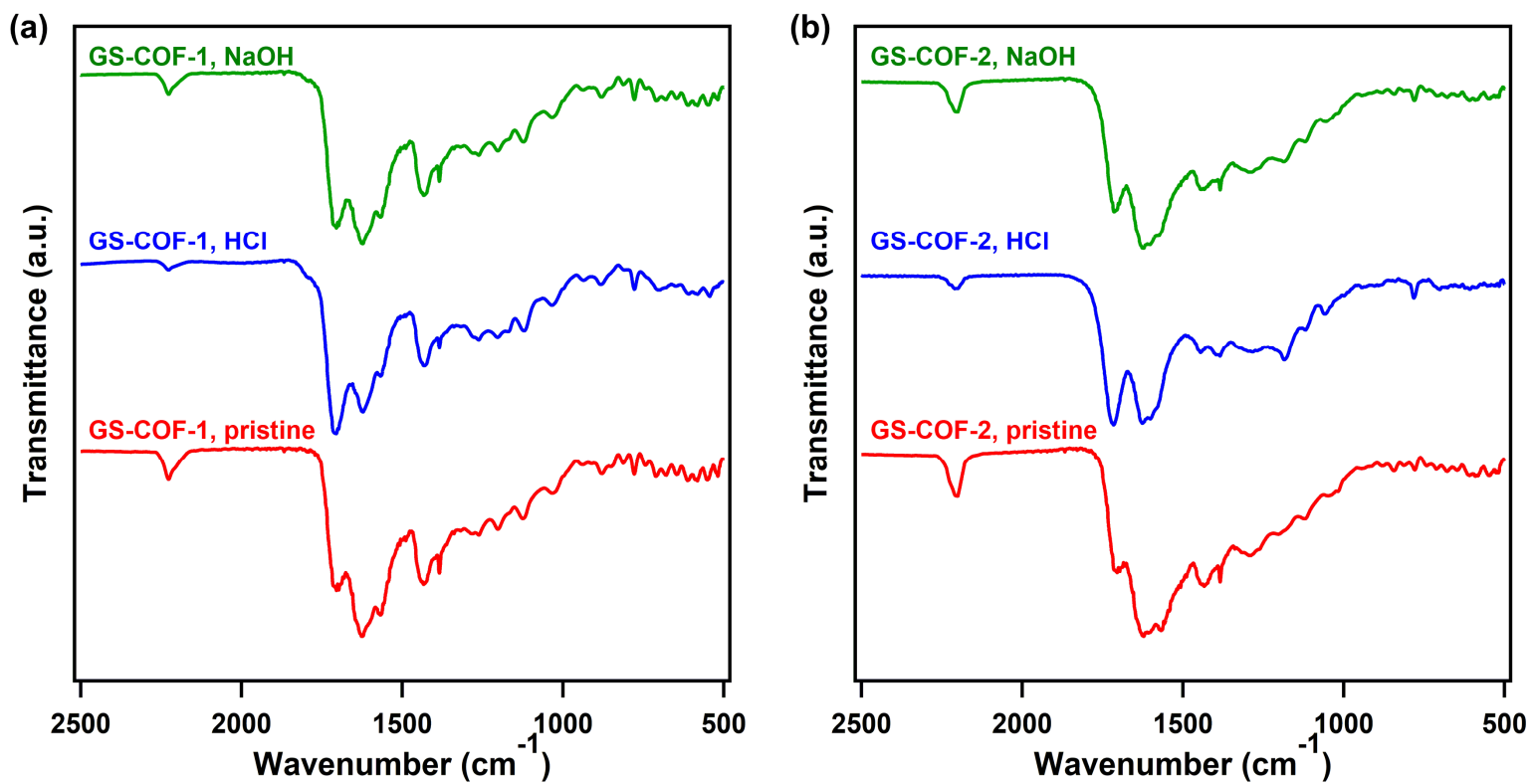

Figure S13. Comparison of FT-IR spectra of pristine, $6 \mathrm{M} \mathrm{HCl}$ and $6 \mathrm{M} \mathrm{NaOH}$ treated (a) GS-COF-1 and (b) -2 . 


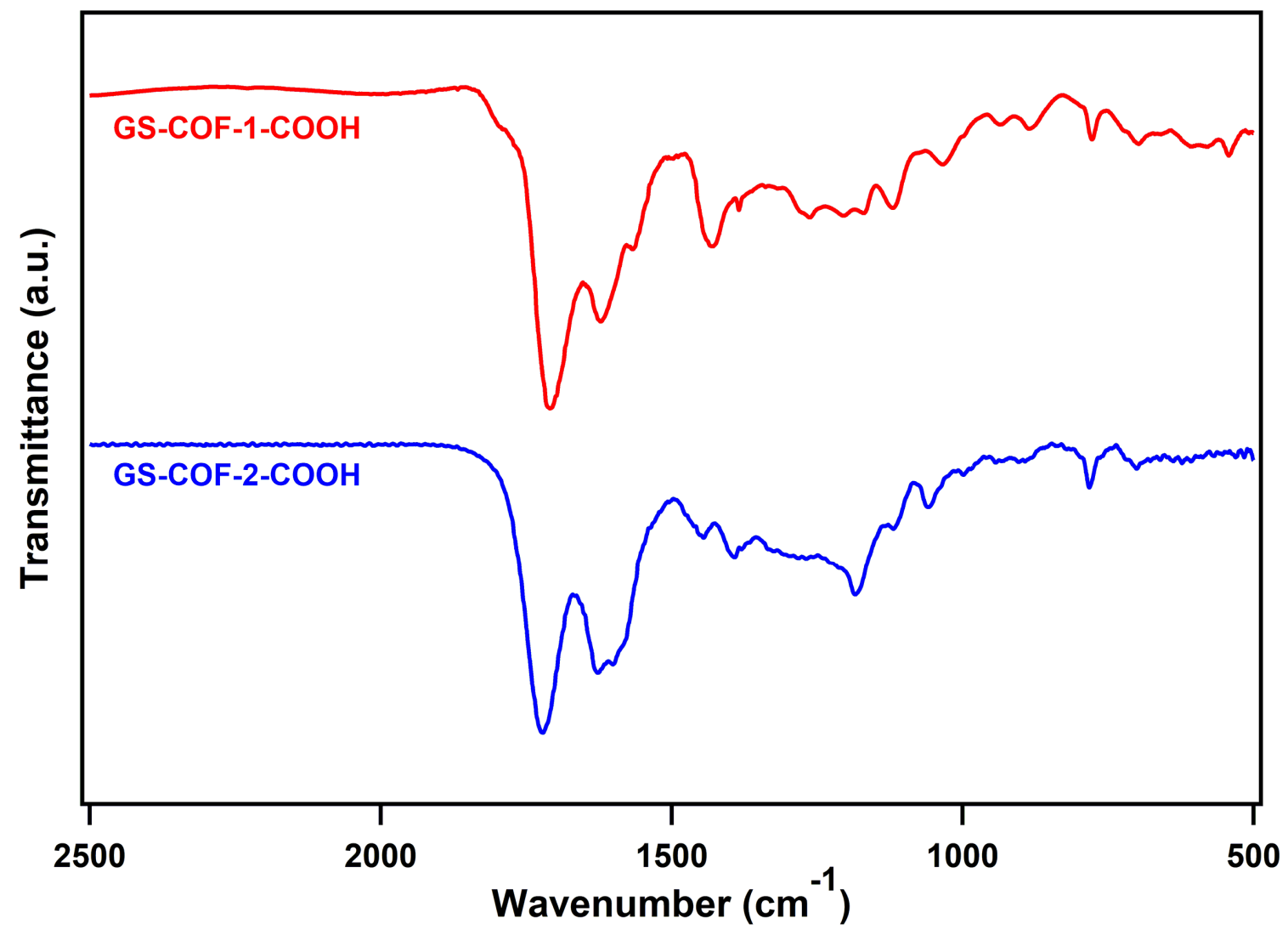

Figure S14. FT-IR spectra of GS-COF-1-COOH and GS-COF-2-COOH, respectively. The complete disappearance of cyano groups demonstrating the full conversion to carboxylic acids. 


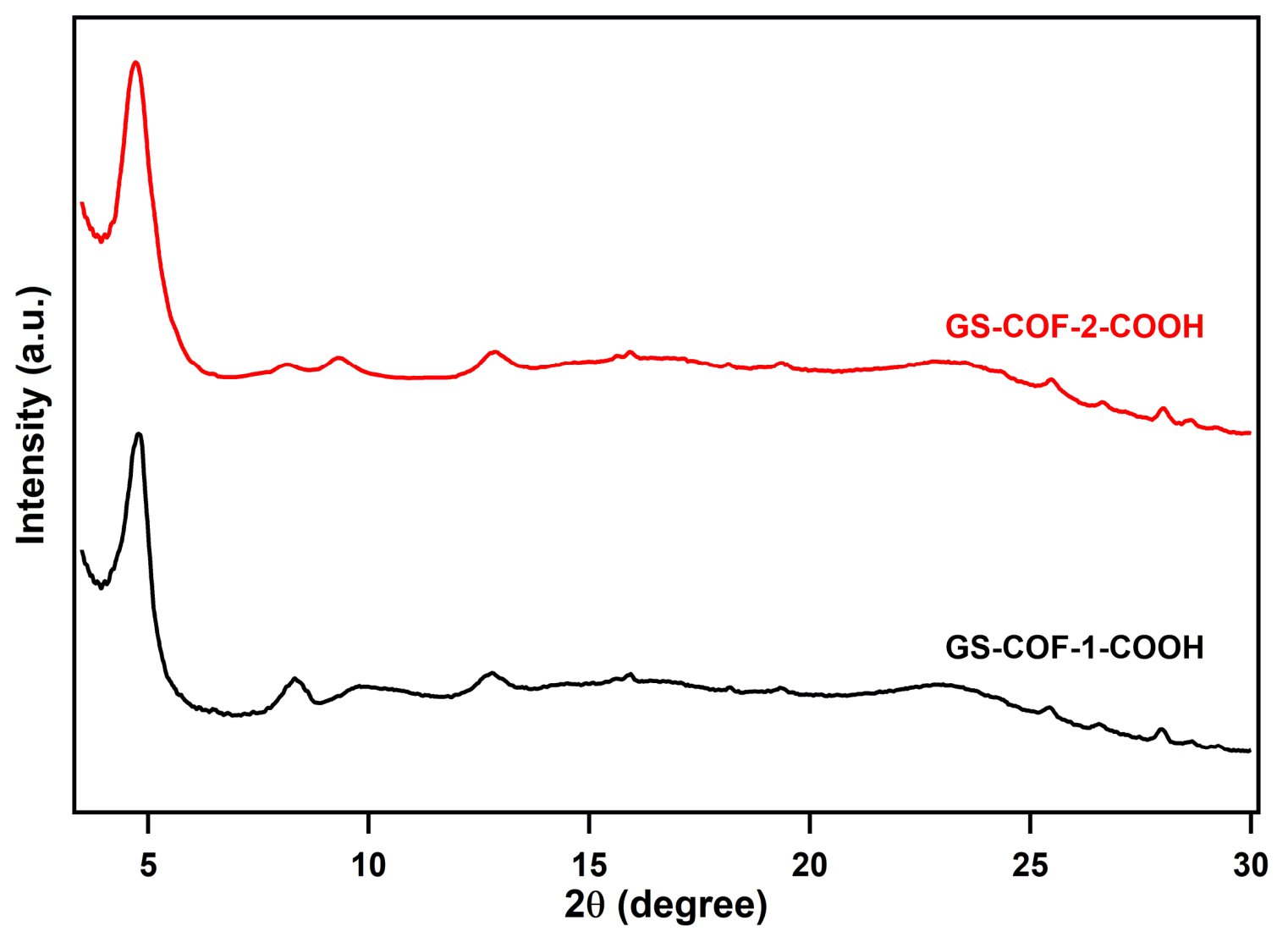

Figure S15. PXRD patterns of GS-COF-1-COOH and GS-COF-2-COOH, respectively. 

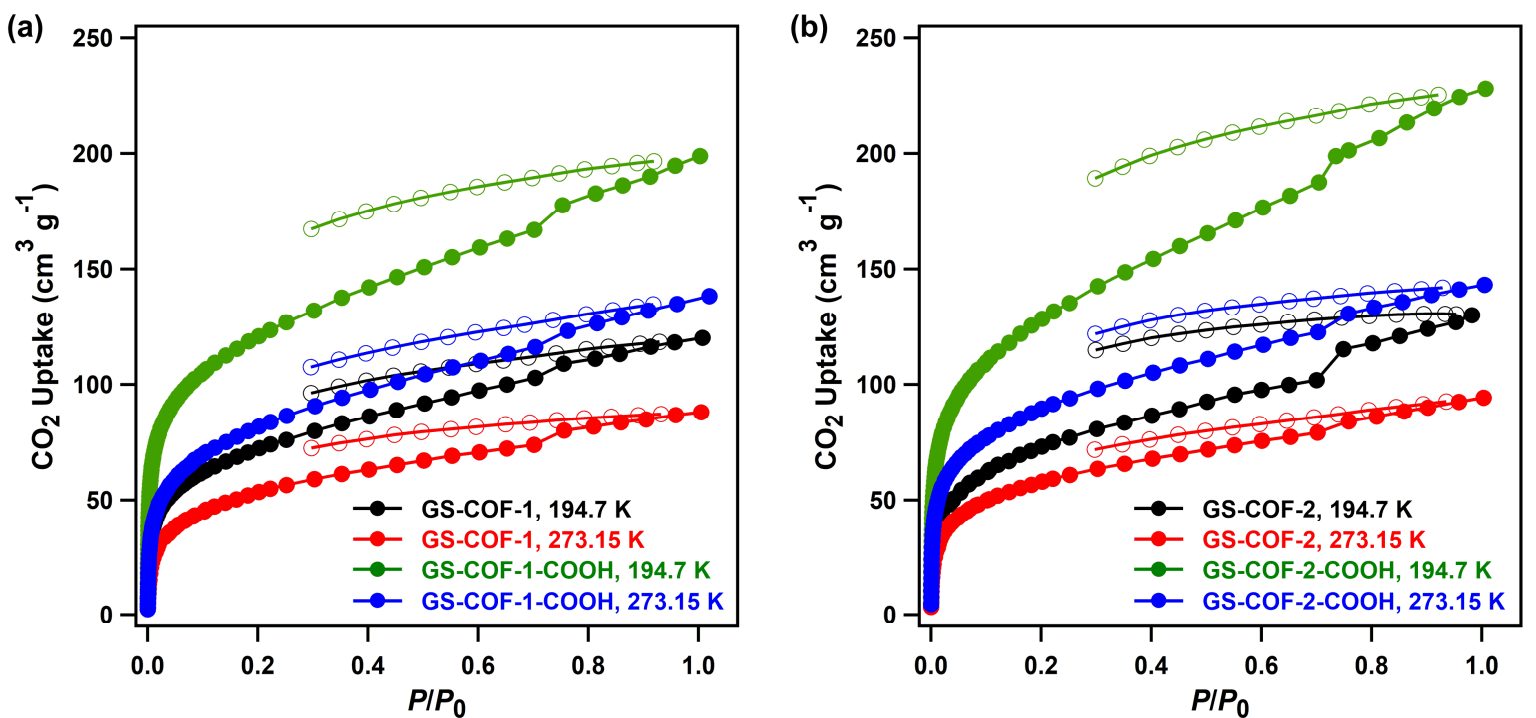

Figure S16. (a) $\mathrm{CO}_{2}$ adsorption isotherms of GS-COF-1 and GS-COF-1-COOH at 194.7 and $273.15 \mathrm{~K}$, respectively. (b) $\mathrm{CO}_{2}$ adsorption isotherms of GS-COF-2 and GS-COF-2-COOH at 194.7 and $273.15 \mathrm{~K}$, respectively. 

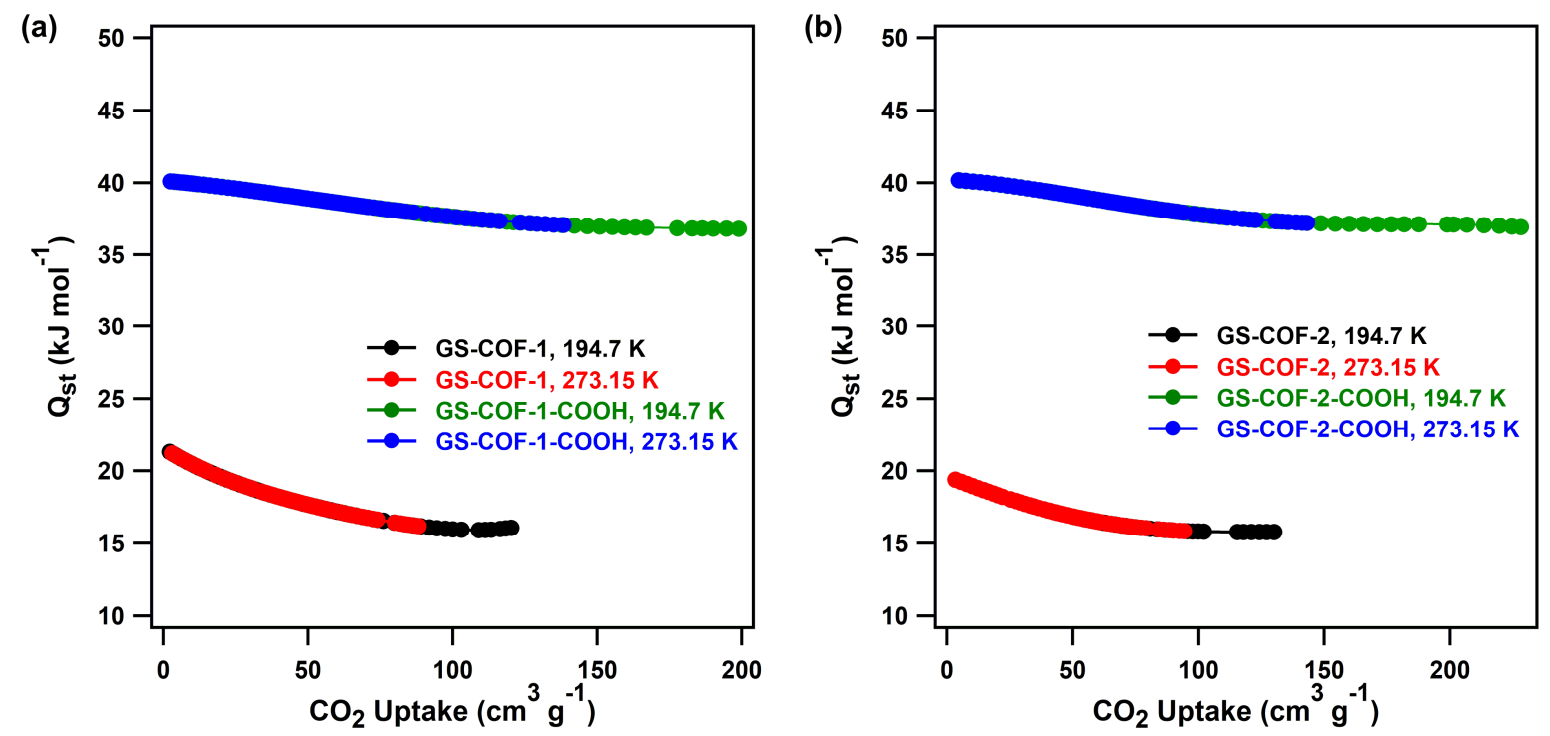

Figure S17. (a) $\mathrm{Q}_{\mathrm{st}}$ curves of $\mathrm{CO}_{2}$ adsorption in GS-COF-1 and GS-COF-1-COOH at 194.7 and $273.15 \mathrm{~K}$, respectively. (b) $\mathrm{Q}_{\mathrm{st}}$ curves of $\mathrm{CO}_{2}$ adsorption in GS-COF-2 and GS-COF-2$\mathrm{COOH}$ at 194.7 and $273.15 \mathrm{~K}$, respectively. 

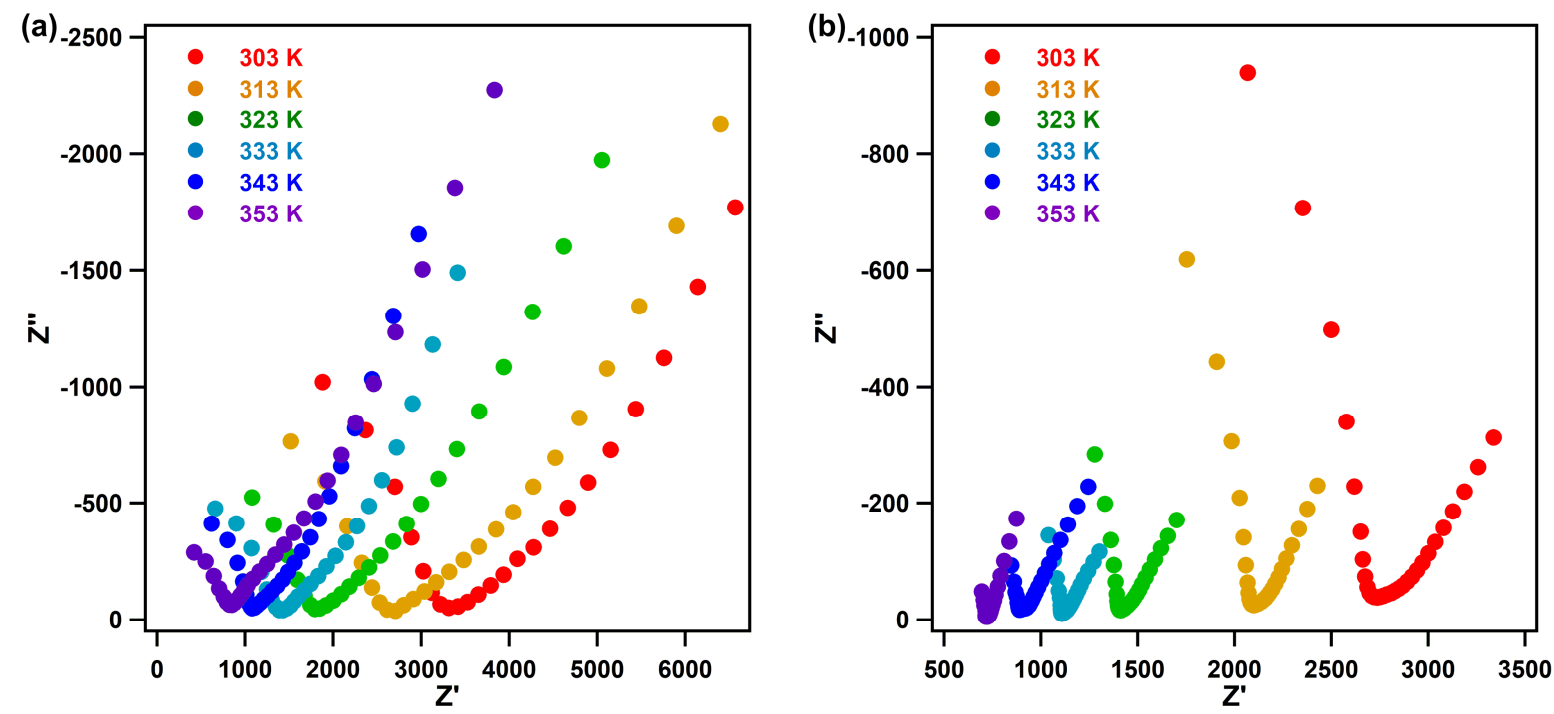

Figure S18. (a) Impedance spectra of GS-COF-1-COOH under $90 \% \mathrm{RH}$ at different temperatures. (b) Impedance spectra of GS-COF-2-COOH under $90 \% \mathrm{RH}$ at different temperatures. 


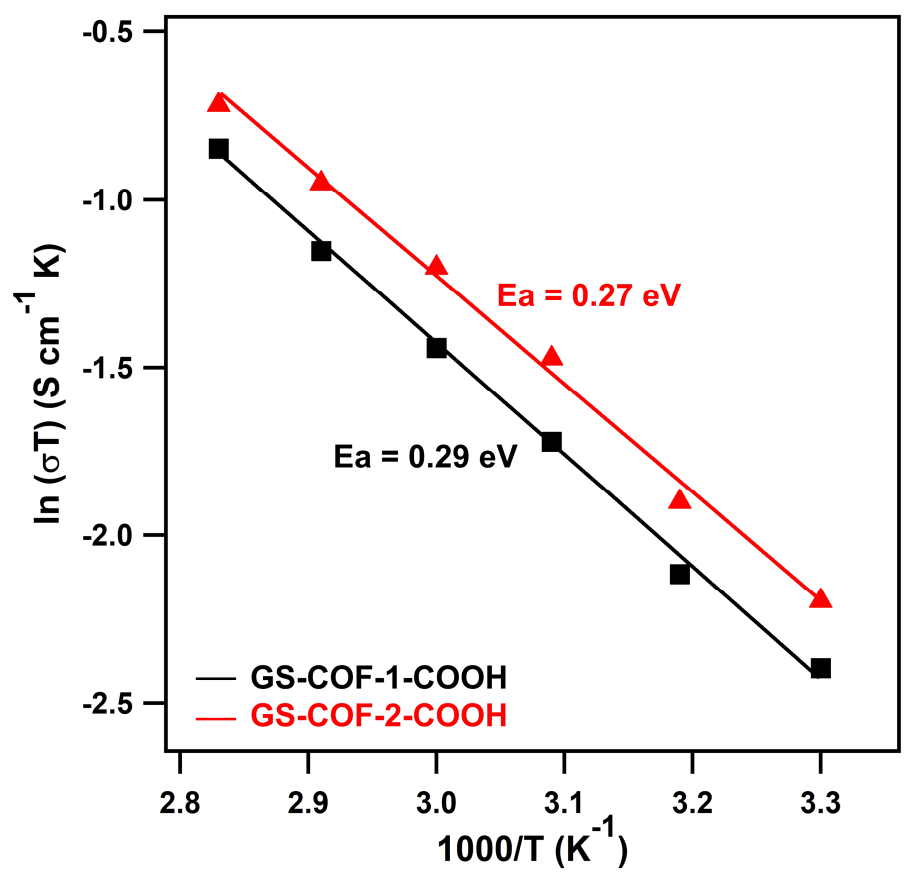

Figure S19. Arrhenius plots for GS-COF-1-COOH (black squares) and GS-COF-2-COOH (red up-triangles). The lines are curve-fitting results. 


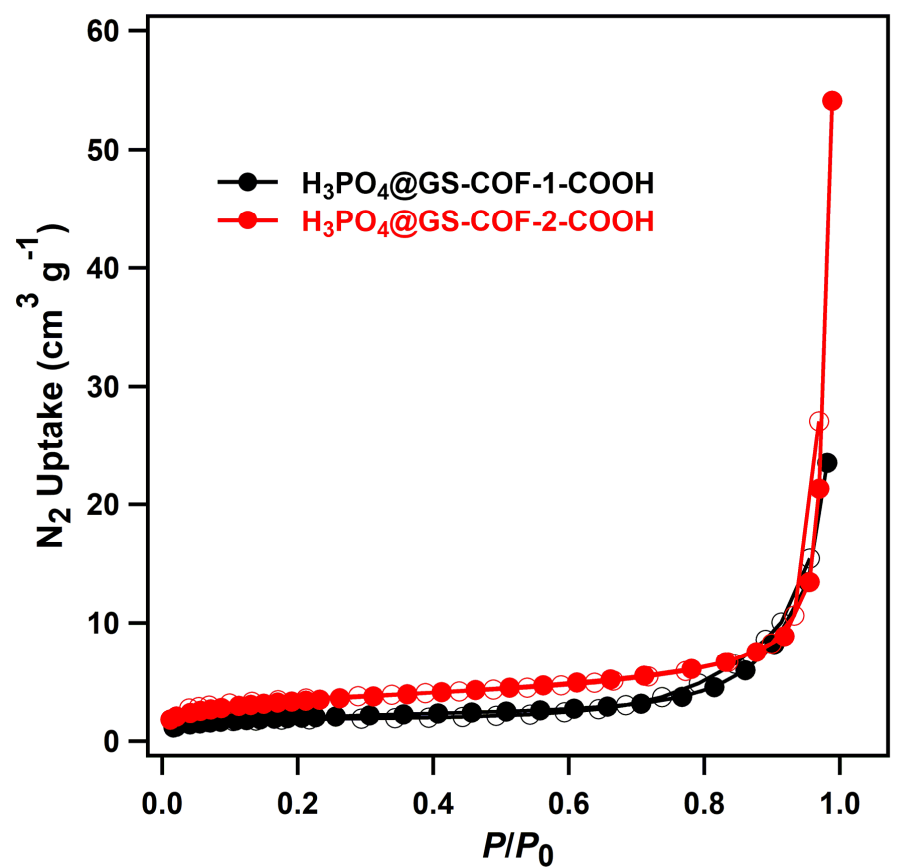

Figure S20. $\mathrm{N}_{2}$ sorption isotherms of $\mathrm{H}_{3} \mathrm{PO}_{4} @$ GS-COF-1-COOH and $\mathrm{H}_{3} \mathrm{PO}_{4} @$ GS-COF-2$\mathrm{COOH}$. Solid and open circles represent the adsorption and desorption branches, respectively. 

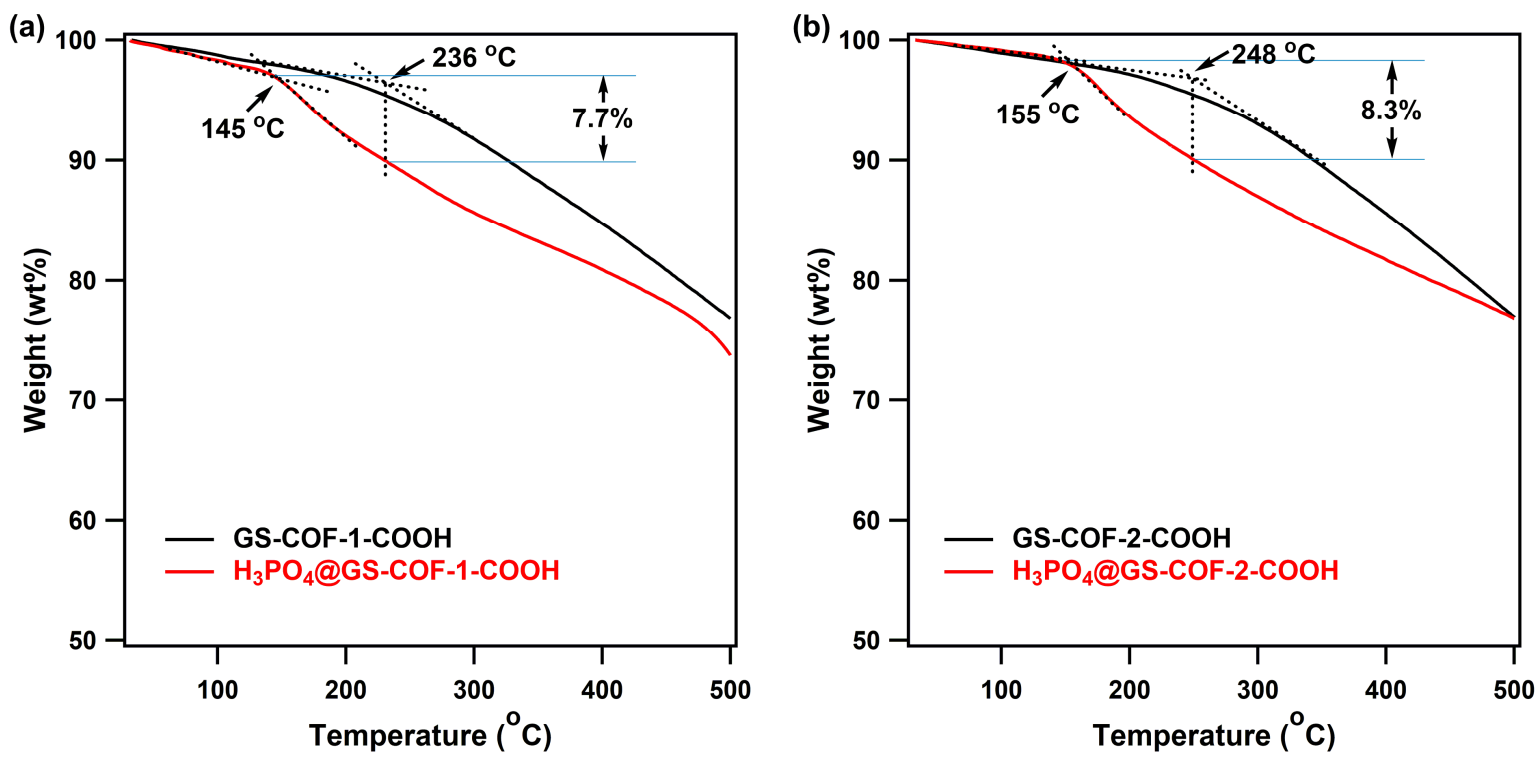

Figure S21. TG curves of (a) GS-COF-1-COOH and $\mathrm{H}_{3} \mathrm{PO}_{4} @$ GS-COF-1-COOH, and (b) GS-COF-2-COOH and $\mathrm{H}_{3} \mathrm{PO}_{4} @$ GS-COF-2-COOH. The The $\mathrm{H}_{3} \mathrm{PO}_{4}$ loadings were calculated as 7.7 and $8.3 \mathrm{wt} \%$ for $\mathrm{H}_{3} \mathrm{PO}_{4} @$ GS-COF-1-COOH and $\mathrm{H}_{3} \mathrm{PO}_{4} @$ GS-COF-2-COOH, respectively. 


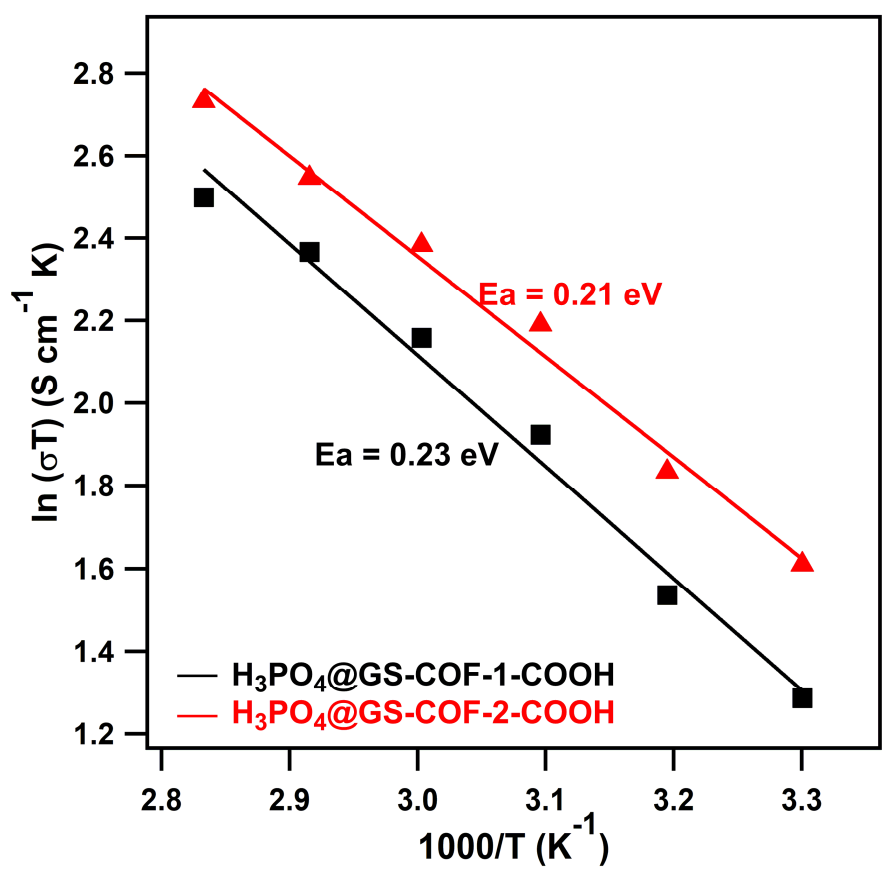

Figure S22. Arrhenius plots for $\mathrm{H}_{3} \mathrm{PO}_{4} @$ GS-COF-1-COOH (black squares) and $\mathrm{H}_{3} \mathrm{PO}_{4} @$ GSCOF-2-COOH (red up-triangles). The lines are curve-fitting results. 


\section{Supporting References}

S1. Sheldrick, G. M. A Short History of SHELX. Acta Crystallogr. Sect. A: Found. Crystallogr. 2008, 64, 112.

S2. Hohenberg, P.; Kohn, W. Inhomogeneous Electron Gas. Phys. Rev. 1964, 136, B864.

S3. Kohn, W.; Sham, L. J. Self-Consistent Equations Including Exchange and Correlation Effects. Phys. Rev. 1965, 140, A1133.

S4. Clark, S. J.; Segall, M. D.; Pickard, C. J.; Hasnip, P. J.; Probert, M. J.; Refson, K.; Payne, M. C. First Principles Methods Using CASTEP. Z. Kristallogr. 2005, 220, 567.

S5. Perdew, J. P.; Burke, K.; Ernzerhof, M. Generalized Gradient Approximation Made Simple. Phys. Rev. Lett. 1996, 77, 3865.

S6. Grimme, S. Accurate Description of van der Waals Complexes by Density Functional Theory Including Empirical Corrections. J. Comput. Chem. 2004, 25, 1463.

S7. Grimme, S. Semiempirical GGA-Type Density Functional Constructed with a LongRange Dispersion Correction. J. Comput. Chem. 2006, 27, 1787.

S8. Tao, S.; Zhai, L.; Wonanke, A. D. D.; Addicoat, M. A.; Jiang, Q.; Jiang, D. Confining $\mathrm{H}_{3} \mathrm{PO}_{4}$ Network in Covalent Organic Frameworks Enables Proton Super Flow. Nat. Commun. 2020, 11, DOI:10.1038/s41467-020-15918-1.

S9. Yang, Y.; He, X.; Zhang, P.; Andaloussi, Y. H.; Zhang, H.; Jiang, Z.; Chen, Y.; Ma, S.; Cheng, P.; Zhang, Z. Predisposed Intrinsic and Extrinsic Proton Conduction in Robust Covalent Organic Frameworks for Hydrogen Fuel Cell Application. Angew. Chem. Int. Ed. 2020, 59, 3678 .

S10. Sasmal, S. H.; Aiyappa, H. B.; Bhange, S. N.; Karak, S.; Halder, A.; Kurungot, S.; Banerjee, R. Superprotonic Conductivity in Flexible Porous Covalent Organic Framework Membranes. Angew. Chem. Int. Ed. 2018, 57, 19804.

S11. Peng, P.; Xu, G.; Hu, Z.; Cheng, Y.; Chi, C.; Yuan, D.; Cheng, H.; Zhao, D. Mechanoassisted Synthesis of Sulfonated Covalent Organic Frameworks with High Intrinsic Proton Conductivity. ACS Appl. Mater. Interfaces 2016, 8, 18505.

S12. Ranjeesh, K. C.; Illathvalappil, R.; Veer, S. D.; Peter, J.; Wakchaure, V. C.; Goudappagouda; Raj, K. V.; Kurungot, S.; Babu, S. S. Imidazole-Linked Crystalline Two-Dimensional Polymer with Ultrahigh Proton-Conductivity. J. Am. Chem. Soc. 2019, $141,14950$.

S13. Meng, Z.; Aykanat, A.; Mirica K. A. Proton Conduction in 2D Aza-Fused Covalent Organic Frameworks. Chem. Mater. 2018, 31, 819.

S14. Xu, H.; Tao, S.; Jiang, D. Proton Conduction in Crystalline and Porous Covalent 
Organic Frameworks. Nat. Mater. 2016, 15, 722.

S15. Ma, H.; Liu, B.; Li, B.; Zhang, L.; Li, Y.-G.; Tan, H.-Q.; Zang, H.-Y. Zhu, G. Cationic Covalent Organic Frameworks: A Simple Platform of Anionic Exchange for Porosity Tuning and Proton Conduction. J. Am. Chem. Soc. 2016, 138, 5897.

S16. Shinde, D. B.; Aiyappa, H. B.; Bhadra, M.; Biswal, B. P.; Wadge, P.; Kandambeth, S.; Garai, B.; Kundu, T.; Kurungot, S.; Banerjee, R. A Mechanochemically Synthesized Covalent Organic Framework as a Proton-Conducting Solid Electrolyte. J. Mater. Chem. A 2016, 4, 2682.

S17. Chandra, S.; Kundu, T.; Kandambeth, S.; BabaRao, R.; Marathe, Y.; Kunjir, S. M.; Banerjee, R. Phosphoric Acid Loaded Azo $(-\mathrm{N}=\mathrm{N}-)$ Based Covalent Organic Framework for Proton Conduction. J. Am. Chem. Soc. 2014, 136, 6570.

S18. Chandra, S.; Kundu, T.; Dey, K.; Addicoat, M.; Heine, T.; Banerjee, R. Interplaying Intrinsic and Extrinsic Proton Conductivities in Covalent Organic Frameworks. Chem. Mater. 2016, 28, 1489.

S19. Xie, Z.; Wang, B.; Yang, Z.; Yang, X.; Yu, X.; Xing, G.; Zhang, Y.; Chen, L. Stable 2D Heteroporous Covalent Organic Frameworks for Efficient Ionic Conduction. Angew. Chem. Int. Ed. 2019, 58, 15742.

S20. Montoro, C.; Rodríguez-San-Miguel, D.; Polo, E.; Escudero-Cid, R.; Ruiz-González, M. L.; Navarro, J. A. R.; Ocón, P.; Zamora, F. Ionic Conductivity and Potential Application for Fuel Cell of a Modified Imine-Based Covalent Organic Framework. J. Am. Chem. Soc. 2017, 139, 10079. 